\title{
J004457+4123 (Sharov 21): not a remarkable nova in M 31 but a background quasar with a spectacular UV flare
}

\author{
H. Meusinger ${ }^{1}$, M. Henze ${ }^{2}$, K. Birkle ${ }^{3,4}$, W. Pietsch ${ }^{2}$, B. Williams ${ }^{5}$, D. Hatzidimitriou ${ }^{6,7}$, R. Nesci ${ }^{8}$, H. Mandel $^{4}$, \\ S. Ertel ${ }^{9}$, A. Hinze ${ }^{10}$, and T. Berthold ${ }^{11}$ \\ 1 Thüringer Landessternwarte Tautenburg, Sternwarte 5, 07778 Tautenburg, Germany \\ e-mail: meus@tls-tautenburg.de \\ 2 Max-Planck-Institut für extraterrestrische Physik, Giessenbachstraße, 85748 Garching, Germany \\ 3 Max-Planck-Institut für Astronomie, Königstuhl 17, 69117 Heidelberg, Germany \\ 4 ZAH, Landessternwarte Heidelberg, Königstuhl 12, Universität Heidelberg, 69117 Heidelberg, Germany \\ 5 Department of Astronomy, Box 351 580, University of Washington, Seattle, WA 98195, USA \\ 6 Department of Astrophysics, Astronomy and Mechanics, Faculty of Physics, University of Athens, Panepistimiopolis, \\ 15784 Zografos, Athens, Greece \\ 7 IESL, Foundation for Research and Technology, 71110 Heraklion, Crete, Greece \\ 8 Department of Physics, University of Roma La Sapienza, Rome, Italy \\ 9 Institut für Theor. Physik und Astrophysik, Christian-Albrechts-Universität zu Kiel, Leibnizstraße 15, 24118 Kiel, Germany \\ 10 Astronomisches Institut, Universität Bern, Sidlerstraße 5, 3012 Bern, Switzerland \\ 11 Sternwarte Sonneberg, Sternwartestr. 32, 96515 Sonneberg, Germany
}

Received 22 October 2009 / Accepted 18 December 2009

\section{ABSTRACT}

\begin{abstract}
Aims. We announce the discovery of a quasar behind the disk of M31, which was previously classified as a remarkable nova in our neighbour galaxy. It is shown here to be a quasar with a single strong flare where the UV flux has increased by a factor of $\sim 20$. The present paper is primarily aimed at the remarkable outburst of J004457+4123 (Sharov 21), with the first part focussed on the optical spectroscopy and the improvement in the photometric database.

Methods. We exploited the archives of photographic plates and CCD observations from 15 wide-field telescopes and performed targetted new observations. In the second part, we try to fit the flare by models of (1) gravitational microlensing due to a star in M 31 and (2) a tidal disruption event (TDE) of a star close to the supermassive black hole of the quasar.

Results. Both the optical spectrum and the broad band spectral energy distribution of Sharov 21 are shown to be very similar to that of normal, radio-quiet type 1 quasars. We present photometric data covering more than a century and resulting in a long-term light curve that is densely sampled over the past five decades. The variability of the quasar is characterized by a ground state with typical fluctuation amplitudes of $\sim 0.2$ mag around $\bar{B} \sim 20.5$, superimposed by a singular flare of $\sim 2 \mathrm{yr}$ duration (observer frame) with the maximum at 1992.81. The total energy in the flare is at least three orders of magnitudes higher than the radiated energy of the most luminous supernovae, provided that it comes from an intrinsic process and the energy is radiated isotropically. The profile of the flare light curve is asymmetric showing in particular a sudden increase before the maximum, whereas the decreasing part can be roughly approximated by a $t^{-5 / 3}$ power law. Both properties appear to support the standard TDE scenario where a $\sim 10 M_{\odot}$ giant star was shredded in the tidal field of a $\sim 2 \ldots 5 \times 10^{8} M_{\odot}$ black hole. The short fallback time derived from the observed light curve requires an ultra-close encounter where the pericentre of the stellar orbit is deep within the tidal disruption radius. This simple model neglects, however, the influence of the massive accretion disk, as well as general-relativistic effects on the orbit of the tidal debris. Gravitational microlensing probably provides an alternative explanation, although the probability of such a high amplification event is very low.
\end{abstract}

Key words. quasars: general - quasars: individual: J004457+4123 - galaxies: individual: M 31 - gravitational lensing: micro black hole physics

\section{Introduction}

Temporal variability is one of the most conspicuous properties for several classes of interesting astrophysical objects. Owing to the unprecedented combination of sky coverage and photometric accuracy, discoveries from the Large Synoptic Survey Telescope (LSST), the Panoramic Survey Telescope and Rapid Response System (Pan-STARRS), the Palomar-QUEST (PQ) survey, or the Palomar Transient Factory (PTF) will provide great advances in the understanding of variable processes, especially of rare transient phenomena (Gezari et al. 2008; Strubbe \& Quataert 2009; Quimby et al. 2009). However, given the nature of the problem, the creation of the observational database for investigating the variability on long time scales in the reference frame of the source, as is the case for quasars, takes a long time. This also holds for recurrent events, e.g. novae, with intrinsically shorter time scales where it is necessary to cover also the wide gaps between the single events. Presently, data mining in archives, most notably in the plate archives from large Schmidt telescopes, remains the only approach if the light curves have to cover a time interval of decades in the rest frame, at least for high-redshift quasars.

In the context of the search for and the identification of optically variable star-like sources in the field of the bright 
Local Group spiral galaxy M 31 (Pietsch et al. 2005a; Henze et al. 2008), our interest was pointed toward the apparent nova J004457+4123, originally discovered by Nedialkov et al. (1996) and described in more detail by Sharov et al. (1998). The light curve presented by these authors clearly shows a strong bump with a maximum brightening by more than 3 mag in the year 1992 while the source remained constant both in the 23 years before and the 5 years after. Sharov et al. suggest that it is a "remarkable nova" in M31, but underscore that it "differs dramatically from typical representatives of this class of objects". Following the terminology of these authors (nova 21), we denote the object as Sharov 21 throughout this paper. A possible X-ray counterpart was first discussed by Pietsch et al. (2005b), who searched for supersoft X-ray counterparts of optical novae in M 31 and identified Sharov 21 with the source [PFH2005] 601 of their catalogue of XMM-Newton EPIC X-ray sources (Pietsch et al. 2005a) and with the hard ROSAT source [SHL2001] 306 from the catalogue of Supper et al. (2001). Based on the hardness of the X-ray source and the peculiar optical light curve, Pietsch et al. (2005b) speculate that Sharov 21 "may not be a nova at all". Here we present, for the first time, optical follow-up spectroscopy which reveals Sharov 21 to be a quasar.

From the very beginning of the investigation of active galactic nuclei (AGN), variability is known to be a diagnostic property of this object class and has been successfully used as a criterion for the selection of quasar candidates in a number of studies (e.g., Kron \& Chiu 1981; Majewski et al. 1991; Hawkins \& Véron 1993; Meusinger et al. 2002, 2003; Rengstorf et al. 2004). AGN originally misclassified as variable stars are neither unprecedented nor unexpected. The most famous case is the prototypical blazar BL Lac, discovered by Cuno Hoffmeister in 1930. However, the misclassification of a luminous quasar as a nova is highly remarkable because it indicates a singular, strong outburst which points toward a rare and interesting transient phenomenon.

It has long been understood that the observed flux variations of AGNs hold keys to the structure of the radiation source. The physical mechanisms behind these fluctuations are however still poorly understood. Frequently discussed scenarios for the origin of the observed optical/UV broad-band long-term (nonblazar) variability related to massive or supermassive black holes in galaxy centres include various processes such as instabilities and non-linear oscillations of the accretion disk (Taam \& Lin 1984; Abramovicz et al. 1989; Honma et al. 1991; Kawaguchi et al. 1998), multiple supernovae in the starburst environment (Terlevich et al. 1992; Cid Fernandes et al. 1997), microlensing of the accretion disk or the broad line region by compact foreground objects (Chang \& Refsdal, 1979; Irwin et al. 1989; Hawkins 1993; Schneider 1993; Lewis \& Irwin 1996; Zackrisson et al. 2003), the disruption of a star which passes within the tidal radius of the supermassive black hole (Hills 1975; Rees 1988, 1990; Komossa \& Bade 1999; Komossa \& Meritt 2008; Gezari et al. 2008), star-star collisions in the dense circumnuclear environment (Torricelli-Ciamponi et al. 2000), and interactions of the components in a supermassive binary black hole (Sillanpää et al. 1988; Lehto \& Valtonen 1996; Katz 1997; Liu \& Chen 2007).

The present paper is aimed at the highly peculiar light curve of the quasar Sharov 21 which is worth detailed investigation. We present the optical spectrum and a significantly improved light curve and discuss possible scenarios for the strong outburst. The observations are described in Sect. 2. The spectrum and other basic properties are analysed in Sect. 3. The outburst is the subject of Sect. 4. Two models are discussed in detail: gravitational microlensing and a stellar tidal disruption event; alternative scenarios are briefly summarized as well. Section 5 gives the conclusions. Standard cosmological parameters $H_{0}=71 \mathrm{~km} \mathrm{~s}^{-1} \mathrm{Mpc}^{-1}, \Omega_{\mathrm{m}}=0.27, \Omega_{\Lambda}=0.73$ are used throughout the paper.

\section{Observational data}

\subsection{Spectroscopy}

The optical spectrum was obtained with the Double Imaging Spectrograph (DIS) on the 3.5-m telescope at Apache Point Observatory (APO) in New Mexico, USA during a campaign to follow-up X-ray sources in M 31. Two exposures were taken for a total of $4500 \mathrm{~s}$. For the blue spectral range (3200 to $5500 \AA$ ), the B 400 reflectance grating was used with a dispersion of $1.85 \AA$ per pixel yielding a nominal resolution of about $7 \AA$ in combination with a 1 1.5 entrance slit. The R 300 grating, with a dispersion of $2.26 \AA$ per pixel, gives a resolution of $8 \AA$ for the red part (5000 to $10100 \AA$ ). The spectra were taken at UT 0300 on 2007-11-09. The observing conditions were excellent through the night.

The spectra were reduced, wavelength calibrated, and flux calibrated using the standard IRAF routines (ccdproc, identify, sunsfunc, apextract, apall). Wavelength calibration was performed using HeNeAr lamp exposures taken just before the object exposures, and flux calibration was performed using a spectrum of the spectrophotometric standard star BD+28-4211.

\subsection{Optical photometry: long-term light curve}

The light curve published by Sharov et al. (1998) is based on $B$ band observations taken with four telescopes between 1969.0 and 1997.7 with a good coverage of the outburst phase. The present study is aimed at an extended and better sampled longterm light curve. We exploited several data archives and combined the results with the data available in the literature. In addition, targetted new observations for another 16 epochs in the years 2006 to 2009 were taken with the CCD Schmidt camera of the Tautenburg $2 \mathrm{~m}$ telescope and with the focal reducer camera CAFOS at the $2.2 \mathrm{~m}$ telescope on Calar Alto ${ }^{1}$, Spain. Most of the archival photographic plates were digitized in the frame of the present work using the Tautenburg Plate Scanner (Brunzendorf \& Meusinger, 1999) for the Tautenburg Schmidt plates, the highquality commercial scanner at the Asiago observatory (Barbieri et al. 2003) for the Asiago plates, and the Microtek ScanMaker 9800XL for the Sonneberg astrograph plates. The Calar Alto plates were scanned for the Heidelberg Digitized Astronomical Plates (HDAP) project using a Heidelberger Druckmaschinen Nexscan F4100 professional scanner and are available from the German Astrophysical Virtual Observatory (GAVO) ${ }^{2}$.

A summary of all used observations from the last six decades is given in Table 1 (CFHT = Canada France Hawaii Telescope, INT = Isaac Newton Telescope, WFS = wide-field survey). $N_{\mathrm{t}}$ is the total number of all single exposures, $N_{\mathrm{e}}$ the number of epochs in the light curve where the quasar has been measured. The last column gives the source of the photometric reduction: (1) this work; (2) Vilardell et al. (2006); (3) Monet al al. (2003); (4) Massey et al. (2006); (5) Sharov et al. (1998).

\footnotetext{
1 The Calar Alto Observatory of the Centro Astronómico Hispano Alemán, Almería, Spain, is operated jointly by the Max-Planck-Institut für Astronomie and the Instituto de Astrofísica de Andalucía (CSIC). 2 http://dc.zah.uni-heidelberg.de
} 
Table 1. Observational material for the construction of the light curve.

\begin{tabular}{|c|c|c|c|c|}
\hline Telescope & $N_{\mathrm{t}}$ & $N_{\mathrm{e}}$ & Years & Source \\
\hline \multicolumn{5}{|c|}{ (a) Digitized photographic plates: } \\
\hline Asiago Schmidt & 24 & 8 & 1968.8-1993.1 & (1) \\
\hline Calar Alto Schmidt & 43 & 15 & 1983.0-2000.7 & (1) \\
\hline Calar Alto $1.2 \mathrm{~m}$ & 8 & 5 & $1976.7-1982.6$ & (1) \\
\hline Palomar Schmidt & 4 & 4 & $1948.7-1989.7$ & $(1),(3)$ \\
\hline Sonneberg $40 \mathrm{~cm}$ & 9 & 1 & 1992.2-1992.6 & (1) \\
\hline Tautenburg Schmidt & 362 & 77 & 1961.5-1997.0 & (1) \\
\hline \multicolumn{5}{|l|}{ (b) CCD observations: } \\
\hline Calar Alto $2.2 \mathrm{~m}$ & 3 & 2 & 2008.7-2009.3 & (1) \\
\hline CFHT 3.6 m & 1 & 1 & 1993.8 & (1) \\
\hline INT (WFS) & 5 & 1 & 1998.8 & (1) \\
\hline INT & 522 & 6 & 1999.7-2003.7 & (2) \\
\hline Kitt Peak 4 m & 10 & 2 & $2000.8,2001.7$ & (4) \\
\hline Skinakas $60 \mathrm{~cm}$ & 5 & 1 & 2007.6 & (1) \\
\hline Tautenburg Schmidt & 30 & 14 & 2006.1-2009.7 & (1) \\
\hline \multicolumn{5}{|c|}{ (c) Original data from Sharov et al. (1998): } \\
\hline four other telescopes & $\sim 150$ & 84 & 1969.0-1997.7 & (5) \\
\hline
\end{tabular}

Altogether, the light curve data pool contains more than 1100 single observations from 15 telescopes. Included are the B magnitudes published by Sharov et al. (1998) for 84 epochs, by the Local Group Galaxies Survey (LGGS; Massey et al. 2006) for 2 epochs, and by Vilardell et al. (2006) binned here into 6 epochs. For the other observations, the photometric reduction was done in the frame of the present study. We used the Source Extractor package (Bertin 1996) for object selection, background correction, and relative photometry, and the LGGS catalogue for the photometric calibration. The reduction was performed under ESO MIDAS. Because of the strongly inhomogeneous background across the disk of M 31 (Henze et al. 2008 ), the photometric calibration was done locally on a $8^{\prime} \times 8^{\prime}$ subimage around Sharov 21 where typically $\sim 100 \pm 50$ calibration stars from the LGGS were identified. Note that the magnitudes given by Sharov et al. (1998) have also been derived from standard stars close to the target. The blue magnitude for the Palomar POSS 1 plate (1953-10-09) is taken from the USNOB1.0 catalogue (Monet et al. 2003) whereas the magnitudes given there for the POSS 2 plate do not agree with the impression from the visual inspection of the images. We re-reduced the image cutouts from the Digitized Sky Survey (DSS2) and derived $B=20.73 \pm 0.23$ and $R=19.66 \pm 0.25$. An early deep plate taken with the $1.2 \mathrm{~m}$ Samuel Oschin Telescope on 1948-09-29 is shown in the Hubble Atlas of Galaxies (Sandage 1961). The visual inspection shows that Sharov 21 is detected with $B=20.3 \pm 0.3$.

With very few exceptions the observations were made through filters reproducing the Johnson UBVR system. About $80 \%$ of the data points in the light curve are from observations in the $B$ band. From several pairs of observations, taken at nearly the same epoch but with different filters, we derive relations between colour indices and the $\mathrm{B}$ band magnitude. The results (Fig. 3) are used to obtain $B$ from observations in the other bands. Because quasar variability is usually not achromatic (see Sect. 3), such colour relations are more suitable than single-epoch colour indices. Photographic magnitudes $m_{\mathrm{pg}}$ from blue sensitive emulsions without filter were transformed by $B \sim m_{\mathrm{pg}}+0.1$.

There are two basic limitations with regard to the final set of data. First, with $B>20$ for most of the time, Sharov 21 is too
Table 2. Detection limits on plates taken before 1950.

\begin{tabular}{lllc}
\hline \hline Telescope & Plate & $\begin{array}{l}\text { Date } \\
\text { yyyy-mm-dd }\end{array}$ & $m_{\mathrm{pg}, \mathrm{lim}}$ \\
\hline Bruce astrograph LHK & $23 \mathrm{a}$ & $1900-09-14$ & 18.8 \\
Bruce astrograph LHK & $265 \mathrm{a}$ & $1901-08-18$ & 18.0 \\
Yerkes 24 inch reflector & $?$ & $1901-09-18$ & 19.5 \\
Bruce astrograph LHK & $649 \mathrm{a}$ & $1903-01-15$ & 18.0 \\
Bruce astrograph LHK & $842 \mathrm{a}$ & $1903-09-27$ & 18.0 \\
Bruce astrograph LHK & $1384 \mathrm{a}$ & $1905-12-26$ & 18.0 \\
Waltz reflector LHK & 198 & $1907-11-02$ & 17.5 \\
Waltz reflector LHK & 369 & $1908-08-20$ & 18.3 \\
Waltz reflector LHK & 603 & $1909-10-19$ & 18.5 \\
Waltz reflector LHK & 4484 & $1934-09-17$ & 18.5 \\
Bruce astrograph LHK & $7163 \mathrm{a}$ & $1949-09-20$ & 18.2 \\
\hline
\end{tabular}

faint to be detected in every plate archive. A high fraction of observations yields therefore upper limits only. In other cases the detections are close to the plate limit resulting in relatively large photometric errors. Second, the epochs of the observations are not regularly distributed but show strong clustering. We stacked the images taken with the same telescope within typically several days to a few weeks applying a quality-weighting factor (Froebrich \& Meusinger 2000) to obtain deeper images with improved signal-to-noise ratio. This procedure was not applied however for the outburst phase in 1992 where we are interested in a high temporal resolution.

The final light curve (Fig. 1) comprises magnitudes at 221 detection epochs but still suffers from several gaps. In particular, no data are available for the early rising phase of the outburst between March and August 1992. We checked the Wide Field Plate Datebase ${ }^{3}$ but found no entries for this time. Also the search in the plate archives of the Baldone Schmidt telescope (Alksnis et al. 1998) and of the 100/300 cm Schmidt telescope of the Kvistaberg Observatory revealed no observations of M 31 during that time.

It is useful to check also older historical observations of the Sharov 21 field which are not deep enough to detect the quasar in its faint stage but would allow discovering a previous outburst. Table 2 lists those observations from the $40 / 200 \mathrm{~cm}$ Bruce double-astrograph and the $72 \mathrm{~cm}$ Waltz reflector of the Landessternwarte Heidelberg-Königstuhl (LHK) with $m_{\mathrm{pg}, \lim } \sim$ 18. A reproduction of a plate taken in 1901 with the 24 inch reflector at Yerkes observatory is shown by Hubble (1929); from the visual inspection we estimate a detection threshold $m \sim 19.5$. Each of these observations excludes the occurrence of a flare similar to that of 1992 for at least several tens of days around their dates of exposure.

\section{General properties of Sharov 21}

In Sect. 3.4 below we demonstrate that the previous classification of Sharov 21 as a remarkable nova in M31 has to be rejected. Our optical spectrum, presented below, clearly reveals the source to be a quasar. The most important properties of this quasar are summarized in Table $3 . t_{1 / 2}$ is the time interval for the decline from maximum flux to half the maximum. Remarks: (1) position from LGGS; (2) ground state/maximum; 3 Both the WFPDB version available via VizieR, (Tsvetkov et al.
1997), and the updated version available via the search browser devel-
oped in Sofia (http: //draco. skyarchive. org/search) were used. 


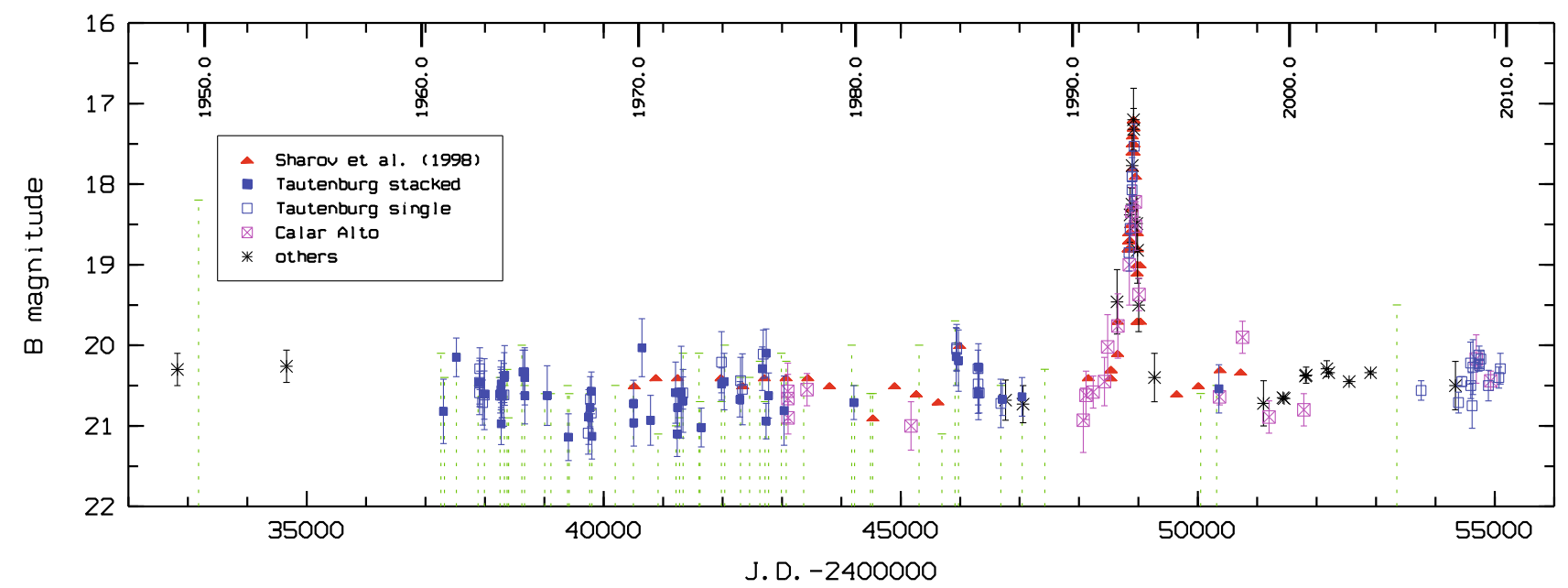

Fig. 1. Long-term B light curve from the data summarized in Table 1 (symbols plus error bars; no photometric errors available for the data from Sharov et al. 1998). Dotted vertical lines: upper limits from images on which the object is not detected. For lucidity, only a small fraction of the upper limit data is shown.

Table 3. Basic properties of Sharov 21 (remarks: see text).

\begin{tabular}{lll}
\hline \hline Measured and derived quantities & & Remark \\
\hline RA $(2000)$ & $00^{\mathrm{h}} 44^{\mathrm{m}} 57^{\mathrm{s}} 94$ & $(1)$ \\
Dec $(2000)$ & $+41^{\circ} 23^{\prime} 43^{\prime \prime} 9$ & $(1)$ \\
redshift $z$ & 2.109 & \\
projected distance from M 31 centre & $26^{\prime}$ & \\
apparent magnitude $B$ & $20.5 / 17.2$ & $(2)$ \\
foreground dust reddening $E(B-V)$ & $0.2 \mathrm{mag}$ & \\
absolute magnitude $M_{\mathrm{B}}$ & $-27.5 /-30.7$ & $(2)$ \\
date of the maximum (year/JD) & $1992.81 / 2448918$ & \\
$t_{1 / 2}$ decline (days) & $15 / 5$ & $(3)$ \\
black hole mass $M_{\text {bh }}$ & $5 \times 10^{8} M_{\odot}$ & $(4)$ \\
$\log \left(L_{\text {bol }} /\right.$ erg s & \\
Eddington ratio $L_{\text {bol }} / L_{\text {edd }}$ & 46.6 & $(5)$ \\
\hline
\end{tabular}

(3) observer frame/quasar rest frame; (4) based on C IV line; (5) mean value for the ground state.

\subsection{Optical light curve and general remarks on variability}

The light curve from the data discussed in Sect. 2 is shown in Fig. 1. Sharov et al. (1998) note that the quasar was "nearly constant from 1969 through 1991 with $B \approx 20.5$ and returned to this value one or two years after the outburst". Compared with the original data from Sharov et al., we (1) basically confirm their finding; (2) extend the covered time interval by about one decade in each time direction; and (3) fill some broad gaps (e.g., between the years 1984 and 1990 and between the beginning of 1993 and 1994). Based on the better sampling of our data, including the upper limits, the possibility of outbursts in intervals that were not covered by observations is thus significantly reduced. Hence, we conclude that the light curve can be divided into (a) the faint state (with $\bar{B}=20.52$ ), which can be considered as the ground state, and (b) a single outburst, or flare, lasting $\sim 2$ yr (JD $2448500 \ldots 2449300$ ) where the quasar was 3.3 mag brighter in the maximum. The flare (Fig. 2) shows a slightly asymmetric profile with three phases: (1) a gradual increase between JD $\sim 248500$ and 2448880 with a gap in the light curve between March and August 1992, followed by (2) an abrupt rise to the maximum at JD $~ 2448918$, and (3) a

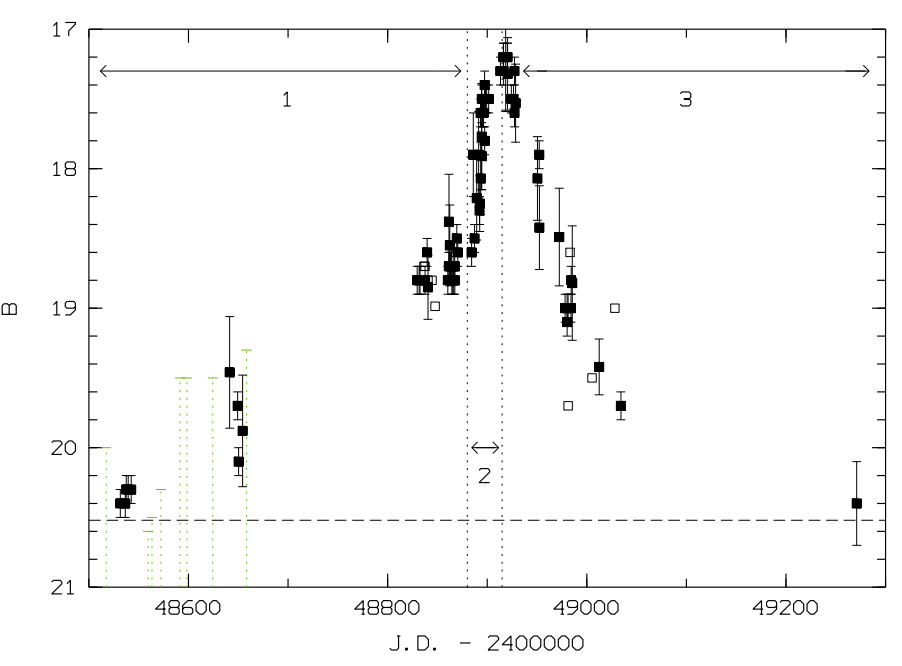

Fig. 2. The light curve in the outburst. Open symbols: uncertain data, dashed lines: upper limits, dotted horizontal line: ground state.

quasi-exponential decline to the ground state at JD 2449300. The interpretation of the outburst will be the subject of Sect. 4 .

To evaluate the variability in the ground state we compare Sharov 21 with quasars from the Tautenburg-Calar Alto Variability and Proper Motion Survey (VPMS; Meusinger et al. 2002, 2003). For Sharov 21 we have a $B$ standard deviation $\sigma_{\mathrm{B}}=0.27 \mathrm{mag}$ from the Tautenburg data $(0.26 \mathrm{mag}$ from all data). For the VPMS quasars with similar redshifts $(z=2.1 \pm 0.2)$ and comparable (extinction-corrected) mean magnitudes $(\bar{B}=$ $19.7 \pm 0.2$ ) we have $\sigma_{B}=0.26 \mathrm{mag}$ in the VPMS field around M 3 (8 quasars) and 0.29 mag in the field around M 92 (6 quasars). We conclude that the flux variability of Sharov 21 , in its ground state, is not unusually strong.

Variations of the $B$ band flux of Sharov 21 are correlated with colour changes. The observed relations (Fig. 3) are qualitatively in agreement with the typical properties of quasars. A hardening of the optical/UV continuum during the bright phase is indicated by multi-frequency monitoring of selected AGNs (Cutri et al. 1985; Edelson et al. 1990; Paltani \& Courvoisier 1994) as well as by statistical studies of AGN ensemble variability (Di Clemente et al. 1996; Cristiani et al. 1997; Trèvese et al. 2001; Vanden Berk et al. 2004). This trend has been confirmed 

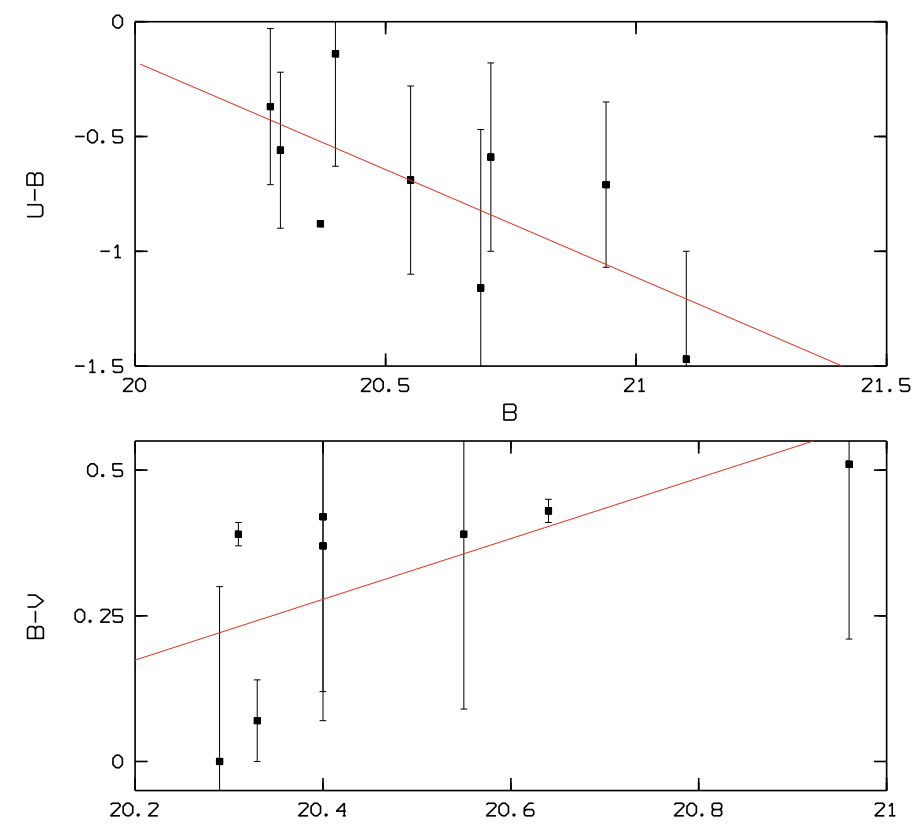

B

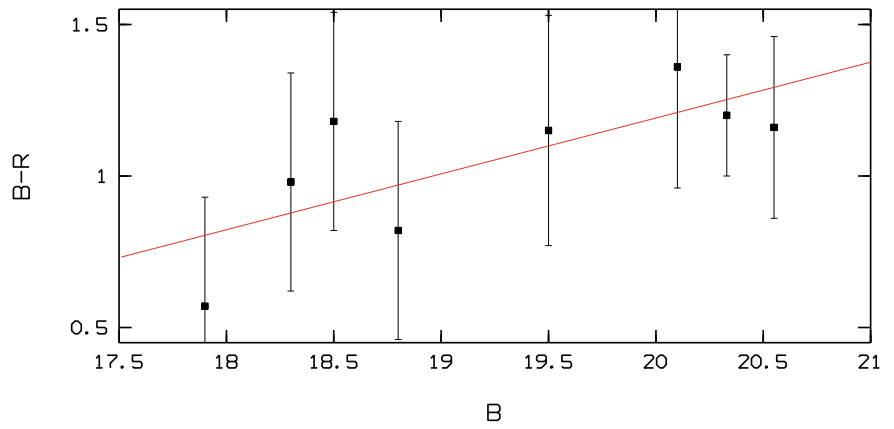

Fig. 3. Colour indices of Sharov 21 as a function of its $B$ magnitude from quasi-simultaneous observations in both bands.

also by multi-epoch spectroscopy of quasars from the Sloan Digital Sky Survey (SDSS) where it was shown that the emission lines are considerably less variable than the continuum, being stronger in the faint stage, relative to the continuum, than in the bright phase (Wilhite et al. 2005). For Sharov 21, the $U$ band is dominated by the strong Lyman $\alpha / \mathrm{N}$ V line (Fig. 5). The contribution of the $\mathrm{C}$ IV line to the flux in the $\mathrm{B}$ band is much smaller, and the other bands are nearly pure continuum. The colour indices $B-V$ and $B-R$ are hence expected to become bluer when the quasar becomes brighter, while $U-B$ becomes redder at the same time. Wilhite et al. present the colour differences between the bright and the faint phase as a function of redshift (their Fig. 14) indicating that $\Delta(u-g)$ has a local minimum at $z \approx 2$ while $\Delta(g-r)$ has a peak at the same redshift. Sesar et al. (2007) found a similar result from the multi-epoch photometric data of quasars in the SDSS stripe 82 with respect to the $g$ and $r$ bands. In an ongoing study of quasar variability in the SDSS stripe 82 (Meusinger et al., in preparation) we confirmed the relation shown by Sesar et al. and derived corresponding relations for the other bands which are in line with the results from Wilhite et al. (2005).

\subsection{Position behind M31}

Figure 4 reveals that Sharov 21 is seen through the disk of M 31 . The ellipses represent the $D_{25}$ isophotes of M31, M 32, and

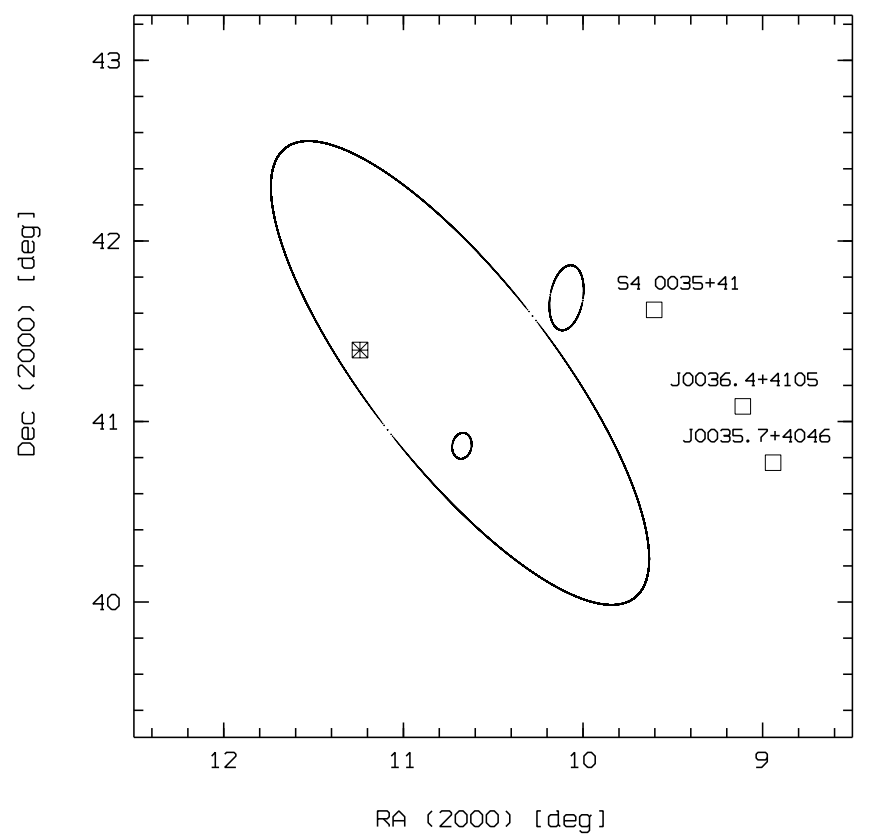

Fig. 4. Map of the known quasars in the M31 field. Open squares: quasars from Véron-Cetty \& Véron (2006), framed asterisk: Sharov 21.

NGC 205, respectively, according to the RC3 (de Vaucouleurs et al. 1991). For a distance of $750 \mathrm{kpc}$ for M 31 (Vilardell et al 2006; their table 1), an inclination angle $i=77^{\circ}$, and a position angle of the major axis of $35^{\circ}$ from the $\mathrm{RC} 3$, its projected distance from the centre of $26^{\prime}(5.7 \mathrm{kpc})$ corresponds to a galactocentric distance of $R=16 \mathrm{kpc}$ in the midplane of M31. A radius of $\sim 30 \mathrm{kpc}$ is a realistic assumption for the extent of the bright disk (e.g. Racine 1991; Ferguson et al. 2002; Irwin et al. 2005). The "Catalogue of Quasars and Active Galactic Nuclei (12th Ed.)" (Véron-Cetty \& Véron 2006) lists only three quasars within $100^{\prime}$ from the centre of M31. However, Sharov 21 is, to our knowledge, the first quasar detected behind the disk of M 31.

\subsection{Foreground reddening}

A foreground spiral galaxy is expected to produce a substantial reddening of a background quasar (Östman et al. 2006). The "Galactic Dust Extinction Service"4 of the NASA/IPAC Infrared Science Archive, which is based on the method pioneered by Schlegel et al. (1998), provides $E(B-V)=0.20$ mag for the position of Sharov 21. Individual reddening values for a large set of globular clusters in M 31 were derived by Barmby et al. (2000) and Fan et al. (2008) yielding $E(B-V)=0.12 \pm 0.03$ mag for the six clusters within $6^{\prime}$ from Sharov 21. The reddening of the quasar must be stronger since only a fraction of the clusters is expected to be located behind the disk of M 31. Finally, a simple model for the radial dependence of the extinction in M31 derived by Hatano et al (1997) yields $A_{B}=0.68$; i.e., $E(B-V)=0.17 \mathrm{mag}$ for the standard Milky Way extinction curve (Savage \& Mathis 1979), which seems to be valid also for M 31 (Barmby 2000). Here we adopt $E(B-V)=0.20$ mag for the total foreground reddening of Sharov 21.

${ }^{4}$ http://irsa.ipac.caltech.edu/applocations/DUST/ 


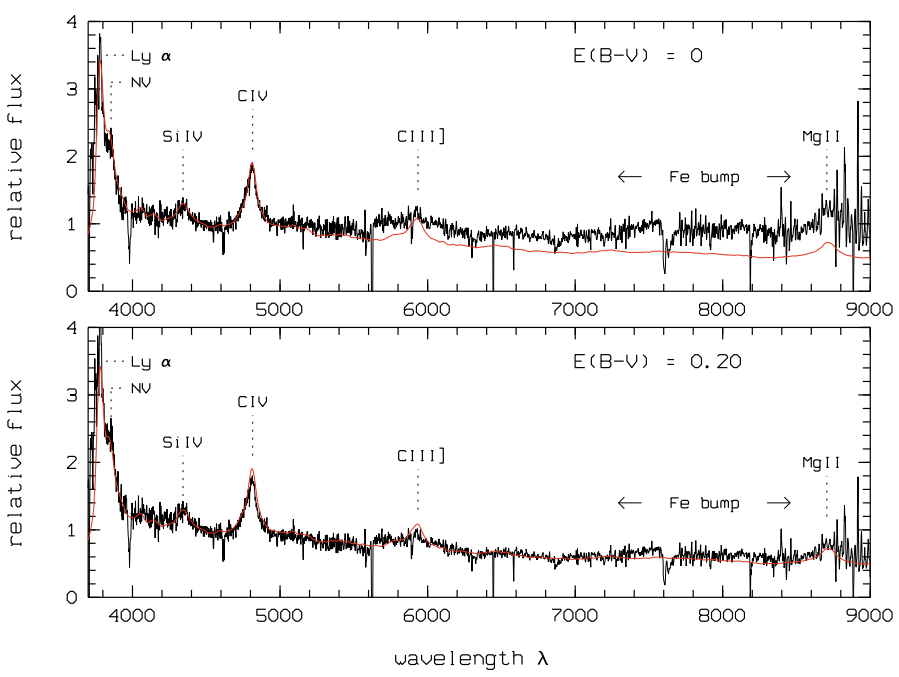

Fig. 5. Observed (top) and foreground extinction-corrected (bottom) optical spectrum of Sharov 21 (observer frame), not corrected for telluric absorption.

\subsection{Optical spectrum}

The optical spectrum is shown in Fig. 5. For comparison the composite spectrum of "normal" quasars from the Sloan Digital Sky Survey (SDSS; Vanden Berk et al. 2001) is plotted (thin smooth curve) shifted to the redshift of Sharov 21, the spectra are normalized at $\lambda 4500 \AA$. We derived a redshift of $z=2.109$ both from the fit of the SDSS composite and directly from the wavelengths of the narrow components of the Lyman $\alpha$ and $\mathrm{N} \mathrm{V}$ lines. Compared with the SDSS composite Sharov 21 has a redder continuum. We de-reddened the spectrum for foreground $(z=0)$ extinction adopting the Milky Way extinction curve. Good agreement with the mean SDSS quasar spectrum is found for $E(B-V)=0.2 \mathrm{mag}$ (Fig. 5, bottom), which is perfectly in line with the reddening value from the NASA/IPAC Infrared Science Archive (see above).

The de-reddened spectrum of Sharov 21 is that of a typical type 1 quasar. There is no evidence of unusual spectral features indicating a peculiar nature of Sharov 21. Compared with the SDSS composite the reddening corrected spectrum shows a stronger Fe bump at $\lambda \sim 7300 \ldots 8300 \AA$ (observer frame) which points towards a relatively high Eddington ratio (Dong et al. 2009). The C III] $\lambda 1909 \AA$ line appears slightly weaker, but note that the line coincides with the Na I foreground absorption at $\lambda \lambda 5890,5896 \AA$. We notice further a weak unidentified absorption line at the position of the N IV $\lambda 1486 \AA$ line.

\subsection{Other wavelength regimes}

M 31 has been observed at radio wavelengths both as part of larger surveys and as the focus of dedicated programmes (see e.g., Gelfand et al. 2004; their Table 1). In no case, a radio counterpart was detected at the position of Sharov 21. With a flux density limit of $0.15 \mathrm{mJy}$, the VLA survey by Braun (1990) is the deepest one at $20 \mathrm{~cm}$ continuum $(1.465 \mathrm{GHz})$. At lower radio frequencies a deep survey of the M31 field was performed by Gelfand et al. (2004, 2005) with the VLA yielding a flux density limit of $3 \mathrm{mJy}$ at $325 \mathrm{MHz}$. Within $d \sim 2^{\prime}$ from Sharov 21, the $325 \mathrm{MHz}$ catalogue lists the sources GLG 043, 045, 050, and 051 . However, with $d>1^{\prime}$ all four radio sources are obviously not related to Sharov 21. The non-detection in the Braun survey

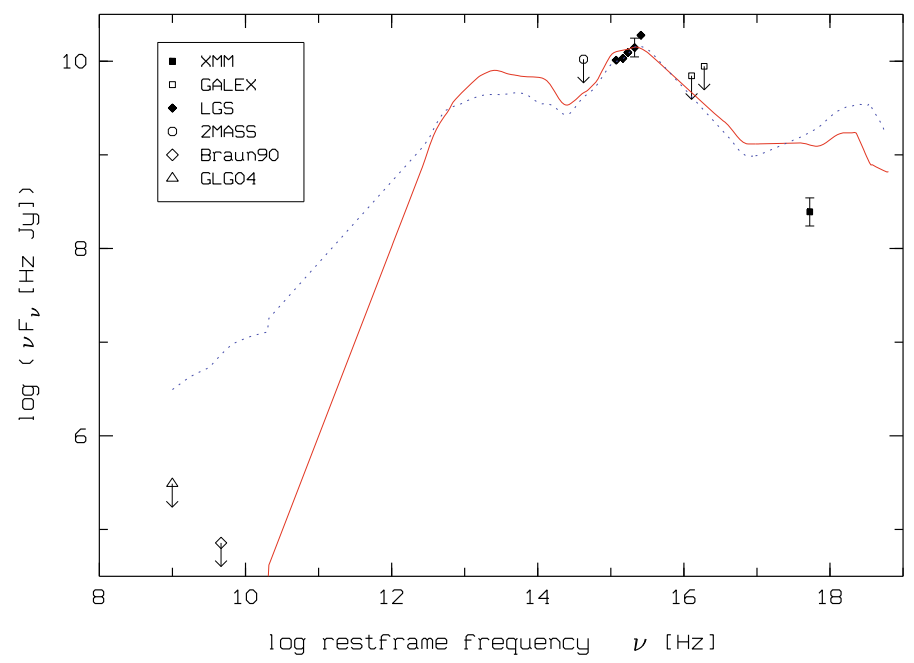

Fig. 6. SED of Sharov 21 in the restframe (open symbols with downward arrows: upper limits). For comparison the mean SED (Elvis et al. 1994) is shown for radio-quiet (solid) and radio-loud quasars (dotted), normalized at $\lambda 1415 \AA$.

implies an upper limit for the radio-loudness parameter $R$, i.e. the ratio of the $5 \mathrm{GHz}$ radio flux density to the $2500 \AA$ optical flux density in the quasar rest frame (Stocke et al. 1992), $\log R^{*}<0.7$ for a radio spectral index $\alpha_{\mathrm{R}}=-0.3$ and $\log R^{*}<0.9$ for $\alpha_{\mathrm{R}}=$ -0.5 . With the threshold $\log R^{*} \geq 1$ for radio-loud AGNs (e.g., White et al. 2000) Sharov 21 is not a radio-loud flat-spectrum quasar.

We re-analysed the archival XMM-Newton and ROSAT data. For computing fluxes from instrument dependent count rates, we used an absorbed power-law model with a generic photon index of 1.7 (see also Pietsch et al. 2005a). We adopted the foreground extinction of $E(B-V)=0.20 \mathrm{mag}$, derived form the optical data, which translates to a $N_{\mathrm{H}}$ of $1.1 \times$ $10^{21} \mathrm{~cm}^{-2}$ following Predehl et al. (1995). Based on this spectral model, we used the source count rates given by Pietsch et al. (2005a) for [PFH2005] 601 in the XMM-Newton observation 0151580401 (2003-02-06) to estimate an unabsorbed flux of

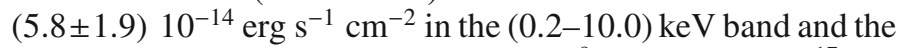
monochromatic flux $F_{v}=(4.5 \pm 1.5) 10^{-9} \mathrm{Jy}$ at $v=1.710^{17} \mathrm{~Hz}$.

The ROSAT data of the Sharov 21 field $^{5}$ consists of 12 observations with the Position Sensitive Proportional Counter (PSPC) and 3 observations with the High Resolution Imager (HRI). The data analysis was done under ESO MIDAS within the EXSAS context. We performed source detection around the position of Sharov 21 on the original event files and computed count rates and $3 \sigma$ upper limits for all observations. There is just one $3 \sigma$ detection of an X-ray source in the data set, identical with [SHL2001] 306, which is supplemented by upper limits for the rest of the observations. The flux estimated from the detection is consistent, within the errors, with the XMM-Newton data. Although the ROSAT observations were performed around JD 2449000 , i.e. during the decline of the UV flare, no significant X-ray variability is detected. Note however, that the only ROSAT detection is a very faint off-axis PSPC detection and due to the large positional error circle of this instrument we can not assume a doubtless correlation with [PFH2005] 601.

We also checked images from the Deep Imaging Survey with the Galaxy Evolution Explorer (GALEX) in the near ultraviolet

\footnotetext{
5 See

http://www . xray.mpe.mpg.de/cgi-bin/rosat/seq-browser
} 
$\left(\lambda_{\text {eff }} \sim 2270 \AA\right)$ and in the far ultraviolet $\left(\lambda_{\text {eff }} \sim 1520 \AA\right)$, but no counterpart could be identified within a radius of $\sim 5^{\prime \prime}$. For detections in the near infrared we searched in the 2MASS catalogue (Skrutskie et al. 2006), again without a clear-cut identification of a counterpart. From simple statistics of the sources around the position of Sharov 21 a flux limit of $1 \mu \mathrm{Jy}$ is estimated for the ultraviolet bands and of $0.25 \mathrm{mJy}$ in the K band. In Fig. 6 we compare the extinction-corrected broad band spectral energy distribution (SED) of Sharov 21 with the mean SED for normal, nonblazar quasars from Elvis et al. (1994).

\subsection{Black hole mass and Eddington ratio}

The black hole mass is estimated from the C IV line width as a measure of proxy for the velocity dispersion of the broad emission line gas in combination with a radius-luminosity $(R-L)$ relationship for the emission region. Significant progress has been achieved over the last years with the calibration of the $R-L$ relation (Vestergaard 2002, 2009; Corbett et al. 2003; Warner et al. 2003; Peterson et al. 2004; Vestergaard \& Peterson 2006).

We use equation (8) from Vestergaard \& Peterson (2006) which is based on the line dispersion $\sigma_{\text {line }}(\mathrm{C}$ IV $)$ and the monochromatic continuum luminosity $\lambda L_{\lambda}$ at $1350 \AA$. Following the method outlined by Peterson et al. (2004) and Vestergaard \& Peterson (2006) we obtain $\sigma_{\text {line }}(\mathrm{C}$ IV $)=(2.9 \pm$ 0.5) $10^{3} \mathrm{~km} \mathrm{~s}^{-1}$. The luminosity of the continuum at $\lambda=1350 \AA$ (restframe) is derived from the extinction-corrected mean $\mathrm{B}$ band flux in the ground state after correction for the contribution from the emission lines $(\sim 12 \%)$ and assuming a standard power-law continuum $F_{\lambda} \propto \lambda^{-(2+\alpha)}$ with $\alpha=-0.5$. We find $\lambda L_{\lambda}(1350 \AA)=1.0710^{46} \mathrm{erg} \mathrm{s}^{-1}$ which finally yields a black hole mass of $M_{\mathrm{bh}} \sim 5 \times 10^{8} M_{\odot}$. Vestergaard \& Peterson (2006) give a standard deviation of \pm 0.33 dex for the scaling relation. Allowing further for the uncertainties of the line width and of the continuum luminosity, the total uncertainty of $M_{\mathrm{bh}}$ is roughly a factor of 3 .

Adopting the bolometric correction $k_{\mathrm{bc}}(1350 \AA)=4$ from Richards et al. (2006; their Fig. 12), the monochromatic luminosity from above corresponds to $L_{\mathrm{bol}}=\lambda L_{\lambda} \cdot k_{\mathrm{bc}}=$ $4.3 \times 10^{46} \mathrm{erg} \mathrm{s}^{-1} \sim 10^{13} L_{\odot}$ and to an Eddington ratio $\epsilon \equiv$ $L_{\mathrm{bol}} / L_{\mathrm{edd}}=3.3 \times 10^{8}\left(M_{\mathrm{bh}} / M_{\odot}\right)^{-1} \sim 0.6$ for the ground state. The overwhelming majority of SDSS quasars with $z \sim 2$ radiate at between $10 \%$ and $100 \%$ of their Eddington luminosity (see Vestergaard 2009) with a narrow $\epsilon$ interval at high luminosities (Kollmeier et al. 2006; Shen et al. 2008; Onken and Kollmeier 2008). According to Onken and Kollmeier, quasars with $\log \left(L_{\mathrm{bol}} / \mathrm{erg} \mathrm{s}^{-1}\right)>46.5$ and $z=1.1 \ldots 2.2$ are characterized by $\langle\log \epsilon\rangle=-0.65 \pm 0.35$ whereas $\epsilon$ is smaller and shows a broader distribution for low-luminosity AGNs. Gavignaud et al. (2008) find $\log \epsilon=-0.97+0.28\left[\log \left(L_{\text {bol }} /\right.\right.$ erg s s $\left.\left.^{-1}\right)-45\right]$ over the interval $\log \left(L_{\mathrm{bol}} / L_{\odot}\right)=45 \ldots 48.5$. As can be seen from their Fig. 4, a value of $\epsilon \sim 0.6$ is relatively high but not unusual for quasars of the redshift and luminosity of Sharov 21. However, in the maximum of the outburst, where the $B$ band flux is a factor of $\sim 20$ higher, the luminosity of Sharov 21 corresponds to a highly super-Eddington regime.

It has been questioned for a couple of reasons whether the mass estimates from scaling relations over-predict the black hole masses by about an order of magnitude. For arguments in favour of the mass estimates we refer to the discussion by Vestergaard (2004). In addition we note that a significantly lower black hole mass would require an unusually high Eddington ratio for Sharov 21.
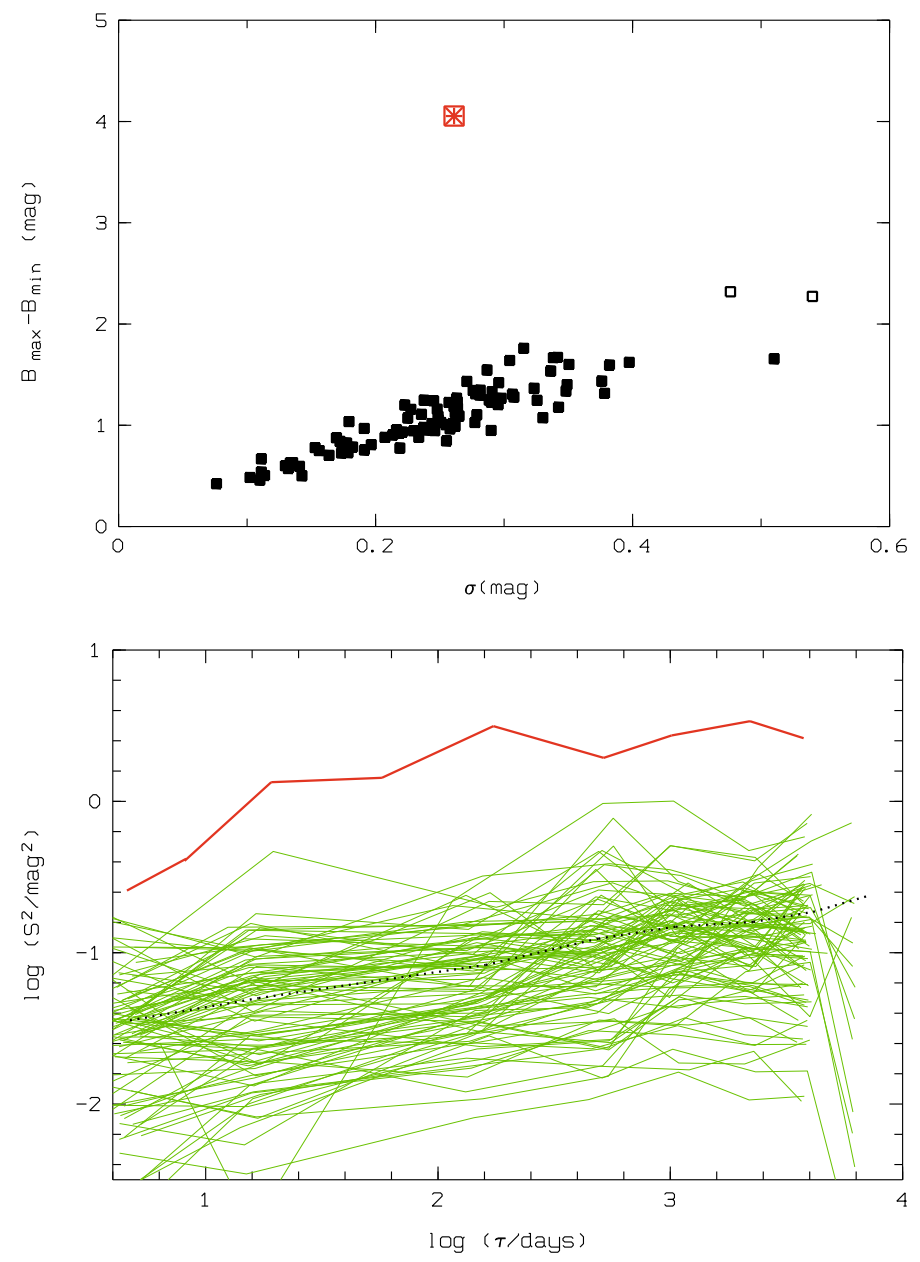

Fig. 7. Comparison of the variability of Sharov 21 with the VPMS quasars. Top: maximum fluctuation $\Delta B_{\max }$ vs. standard deviation $\sigma$ (framed asterisk: Sharov 21; $\sigma$ refers to the ground state). Bottom: single object structure functions for Sharov 21 (solid line at the top) and the VPMS quasars (thin solid lines), and VPMS sample-averaged structure function (dotted line).

\section{The outburst}

\subsection{Sharov21-like UV flares are rare}

UV Flux variations by one or a few magnitudes are observed both in low-luminosity AGNs like NGC 5548 (Ulrich et al. 1997, and references therein) or in blazars (Sect. 4.4). Contrary to the burst of Sharov 21, the $B$ band flux of high redshift radio-quiet quasars typically varies by a few tenths of a magnitude as is observed in the ground state of Sharov 21. The second remarkable difference is the fact that the strong activity of our quasar is limited to a short time interval of $\sim 1$ year (observer frame). During the remaining $\sim 47 \mathrm{yr}$ covered by the light curve (i.e., the ground state), the mean flux variation is a factor of $\sim 16$ smaller than the maximum fluctuation in the outburst. In contrast, both strongly variable low-luminosity AGNs like NGC 5548 and optically violently variable (OVV) quasars show a more or less steady up and down variation.

To get an idea how usual or unusual the outburst of Sharov 21 is, we first consider the light curves of the quasars from the Variability and Proper Motion Survey (VPMS; Meusinger et al. 2002, 2003). The time baseline of the observations is nearly the same (from $\sim 1960$ to 2008) as for Sharov 21, the numbers of observations per quasar are however much lower, typically $\sim 50$. 
The VPMS quasar sample is highly complete down to $B \sim 20$. Among the 321 AGNs in the VPMS there are 10 AGNs with maximum fluctuations $\Delta B_{\max }=B_{\max }-B_{\min } \geq 2 \mathrm{mag}$. For the majority of them $(80 \%)$, the variability is characterized by a more or less monotonic variation over the time baseline. Only for two AGNs the maximum fluctuations can be attributed to burst-like features. One is the strongly variable object CC Boo, a Seyfert galaxy at $z=0.17$ (Margon and Deutsch 1997), the other one is a radio-quiet quasar at $z=1.08$. However, with $\Delta B_{\max }=2.46 \mathrm{mag}$ and $2.50 \mathrm{mag}$, respectively, the burst amplitudes are considerably smaller than for Sharov 21 and there are strong fluctuations also in other parts of the light curves.

In Fig. 7 we compare variability properties of Sharov 21 with those of the VPMS quasars. The top panel shows the maximum fluctuation amplitude versus standard deviation of the B magnitudes in the ground state for Sharov 21, compared with 98 VPMS quasars of comparable redshifts $(z=2.1 \pm 0.5)$. As we cannot reasonably distinguish between a ground state and a higher state for the majority of the quasars we simply use the standard deviation of all data in the light curve as a proxy. (A natural consequence is the increase of $\sigma$ with the maximum amplitude.) The light curves of the two strongly variably VPMS quasars marked by open squares in Fig. 7 are clearly dominated by smooth longterm variations over decades. A popular statistical tool for the investigation of quasar variability is the first order structure function $S^{2}(\tau)=\left\langle[m(t+\tau)-m(t)]^{2}\right\rangle_{\mathrm{t}}$ (e.g., Simonetti et al. 1985; Kawaguchi et al. 1998) where $\tau$ is the time-lag between two observations in the quasar restframe and the angular brackets denote the time-average. The structure function represents a sort of running variance (as a function of the time-lag) and contains therewith information about the time scales of the involved variability processes. The most important conclusion from Fig. 7 is that the flare of Sharov 21 is singular and without comparison in the long-term variability data of the VPMS quasar sample.

Excellent data for the statistical study of quasar variability has been provided from stripe 82 of the Sloan Digital Sky Survey (SDSS) for $\sim 10^{4}$ quasars in five colour bands over $\sim 7$ years (e.g., Sesar et al. 2007). Using the SDSS quasar catalogue (Schneider et al. 2007) we identified 8311 quasars in the Light and Motion Curve Catalogue (LMCC; Bramich et al. 2008) from 249 square degrees of the SDSS stripe 82. No high-redshift quasars with $z>2$ were found with amplitudes in the $\mathrm{u}$ and $\mathrm{g}$ bands $>1.5 \mathrm{mag}$. Allowing for the whole redshift range, the two SDSS quasars with the highest amplitudes in the $\mathrm{g}$ band are SDSS J001130.0+005751.8 $(z=1.49)$ and SDSS J211817.37+001316.8 $(z=0.46)$ with $\Delta g_{\max }=3.2$ and $2.7 \mathrm{mag}$, respectively. Both are bright polarized flat-spectrum radio sources (Jackson et al. 2007; Sowards-Emmerd et al. 2005) and their variability is hence characterized by blazar activity (Sect.4.4). Interestingly, both show (1) a trend of reddening when they become brighter and (2) a trend of increasing intrinsic variability (for definition see Sesar et al. 2007) with increasing wavelength. Such a behaviour is opposite to typical radio-quiet quasars and also to Sharov 21 (Sect. 3.1).

Results from the Palomar-QUEST Survey were recently presented by Bauer et al. (2009). 3113 objects were identified in 7200 square degrees with fluctuation amplitudes $>0.4$ mag on time scales up to $\sim 3.5$ yr. There are only a few objects showing maximum amplitudes $>2$ mag up to $3.7 \mathrm{mag}$; all of them are blazars. Nearly all of the 14800 spectroscopically identified quasars in the data base have jumps $<1 \mathrm{mag}$; the highest value is $1.8 \mathrm{mag}$.

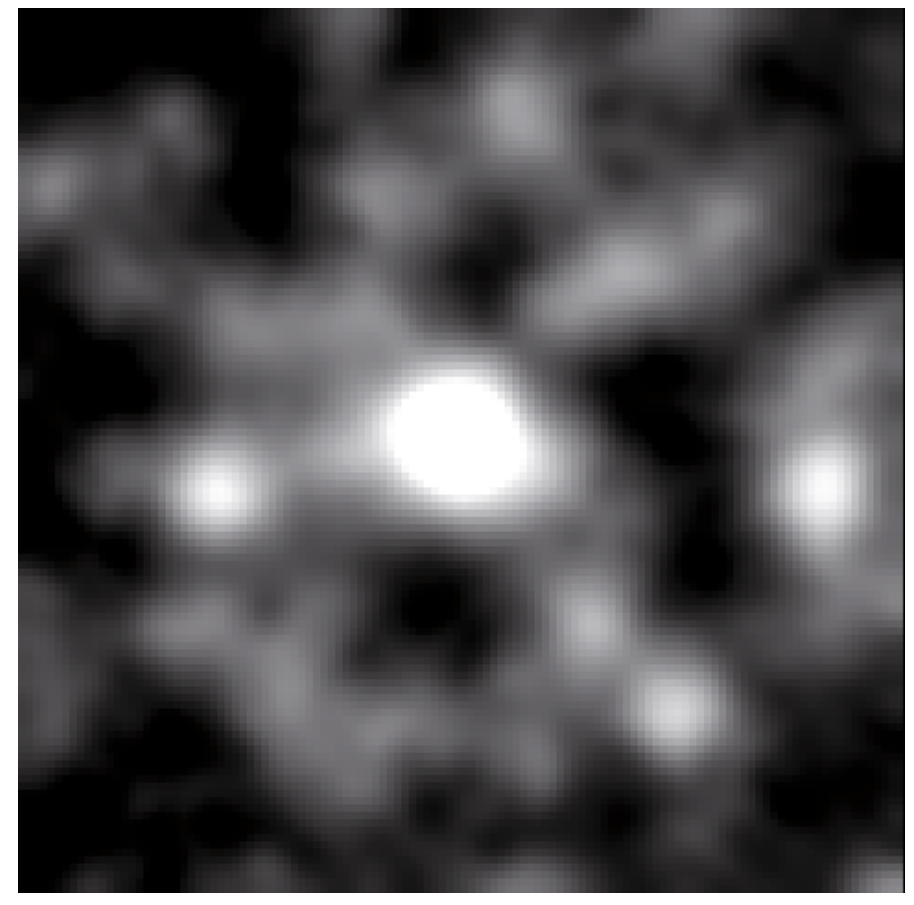

Fig. 8. The crowded field towards Sharov 21 on the $10^{\prime \prime} \times 10^{\prime \prime}$ cutout from the 4-m KPNO image in the $I$ band (N up, E left).

\subsection{A microlensing event?}

As the flare of Sharov 21 appears to be a singular feature in the long light curve, it is tempting to speculate that it originates from a rare event. Here we first discuss microlensing.

Chang and Refsdal (1979) first suggested that the flux of a (macrolensed) quasar can be affected by a star crossing close to the line of sight with time scales of the order of a few months to several years. On the observational side, quasar microlensing was first identified by Irwin et al. (1989). Since then, considerable progress has been made in microlensing simulations, and observations of significant microlensing have been reported in a number of systems (e.g., Pelt et al 1998; Koopmans et al. 2000; Chae et al. 2001; Wisotzki et al. 2003, 2004; Chartas et al. 2004; Eigenbrod et al. 2006; Paraficz 2006; Sluse et al. 2007).

Sharov 21 is seen through M 31, the high star density close to the line of sight is illustrated by Fig. 8. The quasar is the brightest, slightly elongated object in the centre, all other objects are most likely stars in M31. Note also that the quasar appears slightly elongated which points towards an object at a distance $\lesssim 0$ ". 5 . Unfortunately, there are no archival Hubble Space Telescope observations of the field. Extensive imaging was performed with the WFPC2 in 2008 to create an accurate map of M 31 microlensing (PI: A. Crotts) where Sharov 21 is, however, several arcseconds out of the field. A deep image of the field was taken with Subprime-Cam at the $8 \mathrm{~m}$ Subaru telescope in 2004, but the quasar lies exactly in the gap between the two adjacent fields 6 and 7.

From the M 31 mass model (Geehan et al. 2006) we estimate a stellar column density of $\Sigma_{*} \sim 170 M_{\odot} \mathrm{pc}^{-2}$ towards the quasar. This value is $\sim 2.5 \times 10^{-4}$ smaller than the critical surface density $\Sigma_{\text {crit }}=D c^{2} /(4 \pi G)$ with $D=D_{\mathrm{S}} /\left(D_{\mathrm{L}} D_{\mathrm{LS}}\right)$ and $D_{\mathrm{LS}}=D_{\mathrm{S}}-D_{\mathrm{L}}$, where $D_{\mathrm{S}}$ and $D_{\mathrm{L}}$ are the angular distances of the source and the lense, respectively, $G$ is the gravitational constant. The optical depth, i.e. the probability for the quasar to fall into the Einstein radius of a star in $\mathrm{M} 31$, is $\tau=\Sigma_{*} / \Sigma_{\text {crit }} \sim 2.5 \times 10^{-4}$. 
In the case of quasar microlensing by stars in a foreground galaxy with high optical depth the lenses do not act individually and the light curve is complex. For low optical depth $(\tau \lessgtr 0.5)$, however, microlensing can be studied in the single-star approximation (Paczyński 1986), which is applied here. More precisely, the assumptions are made that both the lens and the source are point-like and that the relative motion of the lens is linear. Then the light curve is given by the magnification

$\mu(t) \equiv \frac{F_{v, \mathrm{obs}}(t)}{\bar{F}_{v, \mathrm{gs}}}=\frac{u(t)^{2}+2}{u(t) \sqrt{u(t)^{2}+4}}$,

where $u(t)$ is the angular distance between source and lens in units of the Einstein angle $\Theta_{\mathrm{E}}$, and $F_{v, \mathrm{obs}}(t)$ and $\bar{F}_{v, \mathrm{gs}}$ are the observed monochromatic flux density at time $t$ and the mean flux density in the ground state, respectively, in the $B$ band. As $D_{\mathrm{S}}$ and $D_{\mathrm{L}}$ are given, the light curve depends only on the mass $M_{\mathrm{L}}$, the relative transverse velocity $v_{\mathrm{t}}$ of the lens, and the impact parameter, i.e., the minimum distance $u_{\text {min }}$ between lens and source. The light curve of a high magnification event can be significantly modified by the finite size of the source. This, however, occurs for $u_{\text {min }} \sim R_{*} / R_{\mathrm{E}}$, with $R_{\mathrm{E}}=\Theta_{\mathrm{E}} D_{\mathrm{L}}$, whereas we have $u_{\text {min }} \sim 10^{3} R_{*} / R_{\mathrm{E}}$. Furthermore, for a scale of $8.4 \mathrm{kpc} / "$ at the redshift of Sharov 21 and assuming that the source size of the UV radiation of the quasar is $\lesssim 10^{13} \mathrm{~m}$, the source has an angular diameter about two orders of magnitude smaller than the minimum impact parameter and can be considered as point-like.

The transverse velocity is determined by the motion of the lens in M31, the proper motion of M 31 with respect to the barycentre of the Local Group (LG), the motion of the Sun around the Galactic centre, the motion of the Galaxy around the Local Group barycentre, and the motion of the LG relative to the cosmic microwave background (CMB). Since the first two effects are poorly constrained, we consider here for simplicity only the velocity of the $\mathrm{LG}$ with respect to the CMB. With $v_{\mathrm{LG}-\mathrm{CMB}}=612 \mathrm{~km} \mathrm{~s}^{-1}$ towards $(l, b)=\left(270^{\circ}, 29^{\circ}\right)($ Loeb \& Narayan 2008) we have $v_{\mathrm{t}} \sim 300 \mathrm{~km} \mathrm{~s}^{-1}$.

For the simplifying assumption that all lenses have the same mass and velocity, the Einstein radius crossing time $t_{\mathrm{E}}=\Theta_{\mathrm{E}} / v_{\mathrm{t}}$ is constant and the event rate for quasar microlensing is estimated by $\Gamma \sim 2 N_{\mathrm{q}} \tau /\left(\pi t_{\mathrm{E}}\right)$ (e.g., Mao 2008), where $N_{\mathrm{q}}$ is the number of quasars. Given $\sim 20$ quasars with $B<20$ per square degree and a surface area of $\sim 2$ square degrees within the 25 mag isophote of M 31, we have $N_{\mathrm{q}} \sim 40$ and $\Gamma \sim 1$ per century. Hence, the discovery of a microlensed, faint quasar behind M31 over an interval of half a century is not unlikely.

When the tranverse velocity and the distances are fixed, the maximum amplification is determined by the impact parameter $u_{\min }$ and the time scale $t_{\mathrm{E}}$ is determined by $M_{*}$. For $v_{\mathrm{t}}=300 \mathrm{~km} \mathrm{~s}^{-1}$ the light curve is best fitted with $M_{*} \sim 0.3 M_{\odot}$ and $u_{\text {min }} \sim 0.048$ (Fig. 9, top). A higher velocity requires a higher stellar mass. The fit is not perfect because the observed light curve shows a weak but clearly indicated asymmetry (Fig. 2) which hints on deviations from the single-lens hypothesis. Therefore we add a second lens which moves with the same $v_{\mathrm{t}}$ but crosses the line of sight $\sim 180$ days earlier. For simplicity, each component is treated as a single star, the light curve is computed via the multiplicative magnification approximation (Vietri \& Ostriker 1983) $\mu=\mu_{1} \cdot \mu_{2}$. A good fit to the shoulder in the light curve between 400 and 50 days before the maximum is achieved for $\left(M_{*, 1}, M_{*, 2}\right)=\left(0.3 M_{\odot}, 0.1 M_{\odot}\right)$ if $v_{\mathrm{t}}=300 \mathrm{~km} \mathrm{~s}^{-1}$ and $\left(1.2 M_{\odot}, 0.4 M_{\odot}\right)$ if $v_{\mathrm{t}}=600 \mathrm{~km} \mathrm{~s}^{-1}$, respectively, with $\left(u_{\min , 1}, u_{\min , 2}=0.055,0.8\right)$ in both cases (Fig. 9). The projected linear separation of the two lenses is $\sim 35 \mathrm{AU}$. Both the mass ratio and the separation are not atypical of a binary star.
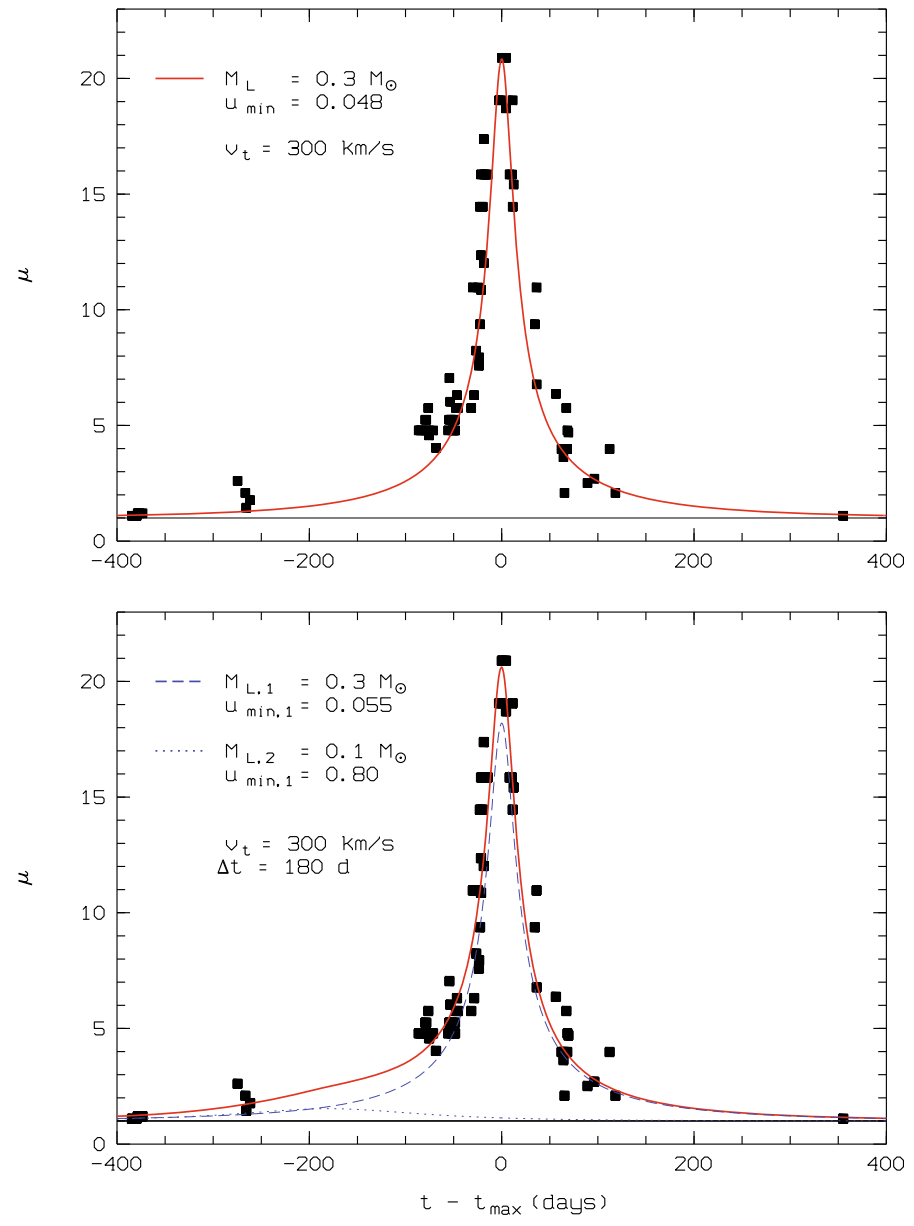

Fig. 9. Light curve $\mu(t)$ of Sharov 21 around its maximum (no error bars for lucidity, open symbols: uncertain data). Overplotted are the fitting curves (solid) for microlensing by one single star (top) and two single stars of the masses $M_{\mathrm{L}, 1}$ and $M_{\mathrm{L}, 2}$ (bottom).

Although microlensing by stars in M 31 appears to be a plausible explanation for the flare of Sharov 21, there are serious objections. First, the probability for magnification as strong as in Sharov 21 is very low. With regard to his model with $\tau=0.1$, Paczyński (1986) points out that "appreciable increases in intensity are there once per millennium ... there is not much hope in detecting intensity changes due to microlensing at such low optical depth". Second, colour index variations are expected in the case of quasar microlensing (Wambsganss \& Paczyński 1991; Yonehara et al. 2008) if the source appears extended. For the point source-point lens constellation, however, an achromatic light curve is expected. Here, the source can be considered pointlike, but $B-R$ was considerably smaller in the flare compared to the faint state (Fig. 3). Third, as the angular separation of the two lenses is $\sim \Theta_{\mathrm{E}, 1}$, the magnification pattern is expected to be more complex then for the single-star approximation. Detailed modelling with a more consistent treatment of the binary lens problem is clearly necessary but is beyond the scope of the present paper. It will be particularly interesting to see if such models find a natural interpretation for the steep rise of the flux before the peak.

\subsection{A stellar tidal disruption event?}

An alternative process which is rare and produces a strong $\mathrm{UV} / \mathrm{X}$-ray flare is the disruption of a star by the strong tidal forces of a massive black hole (Lidskii \& Ozernoi 1979; Rees 1988, 1990). Tidal disruption events (TDEs) were discussed so 
far mainly in the context of dormant black holes in non-AGN galaxies or in low-luminosity AGNs (e.g., Phinney 1989; Rees 1990) and, more recently, of recoiling black holes (Komossa \& Meritt 2008). At least a fraction of low-luminosity AGNs appears to be powered by stellar tidal disruptions (Komossa et al. 2004; Milosavljević et al. 2006). For high-luminosity AGNs the situation is more complicated. TDEs are expected to be rare for black hole masses higher than a critical mass $M_{\mathrm{bh}}>M_{\text {crit }} \approx$ $10^{8} M_{\odot}$ where the gravitation (Schwarzschild) radius, $R_{\mathrm{S}}$, exceeds the tidal disruption radius of solar mass stars so that such stars are swallowed whole without disruption (Hills 1975; see also Chen et al. 2008; Gezari et al. 2008). On the other hand, it seems possible that a massive, self-gravitating accretion disk brings more stars into loss-cone orbits and enhances therefore the tidal disruption rate (Syer et al. 1991; Donley et al. 2002).

Until now, about a dozen TDE candidates in non-AGN galaxies have been found from X-ray surveys (Komossa 2002; Komossa et al. 2009; Esquej 2007, 2008; Cappelluti et al. 2009) and also in the UV/Optical (Renzini et al. 1995; Gezari et al. 2006, 2008). None of the events detected so far were found to be related to a high-luminosity AGN. Such a detection would be interesting because it provides an opportunity to check the basic tidal disruption theory as their predictions generally depend on $M_{\text {bh }}$ which can be estimated independently in this case. However, as noted by Gezari et al. (2008), the existence of various mechanisms for the UV variability of quasars makes the interpretation of a UV flare subject to careful analysis. In particular it is necessary to make sure that the observed flare is not just a more or less usual feature in a strongly variable light curve.

The theoretical frame for the interpretation of TDEs has been set with the pioneering work by Hills (1975), Lacy et al. (1982), Rees (1988), Phinney et al. (1989), Evans \& Kochanek (1989), and more recently by, among others, Magorrian \& Tremaine (1999), Ulmer (1999), Ayal et al. (2000), Ivanov \& Novikov (2001), Menou \& Quataert (2001), Li et al. (2002), Bogdanović et al. (2004), Wang \& Merritt (2004), Chen et al. (2008), Lodato et al. (2008). We start with a short summary of the basic theory. A star of mass $M_{\star}$ and radius $R_{\star}$, originally in hydrostatic equilibrium and on a parabolic orbit, passing at the time $t_{\mathrm{d}}$ within the tidal disruption radius

$R_{\mathrm{t}}=R_{*}\left(M_{\mathrm{bh}} / M_{*}\right)^{1 / 3}$

of the black hole will be torn apart by the tidal forces. The change in the black hole potential over the star will produce a spread in the specific energy of the gas. After the encounter, the gas particles will have a considerably widened energy distribution. Following Rees (1988), about half of the mass has negative energy and is thus gravitationally bound to the black hole, the other half is unbound and will be ejected from the system. The bound elements move in highly excentric elliptical orbits of the size $2 a=R_{\mathrm{p}}+R_{\mathrm{a}} \sim R_{\mathrm{a}}$ where $R_{\mathrm{p}}$ and $R_{\mathrm{a}}$ are the pericentric and the apocentric distance, respectively. With the Keplerian relation between the energy and the size of the orbit and after replacing $a$ by the orbital period $P$ we have $E=-\frac{1}{2}\left(2 \pi G M_{\mathrm{bh}} / P\right)^{2 / 3}$ for $M_{\mathrm{bh}} \gg M_{*}$. After one orbit the bound elements will return to $R_{\mathrm{p}}$ and efficiently loose their energy and angular momentum via stream-stream collision and finally be accreted to the black hole. The consequence is a luminous flare at $t_{0}$ with a peak in the $\mathrm{UV} / \mathrm{X}$-rays and a spectrum which is characterized by a blackbody temperature of a thick disk or a spherical envelope at the tidal radius $R_{\mathrm{t}}$ from Eq. (2):

$T_{\text {eff }}=\left(\frac{L}{4 \pi \sigma R_{*}^{2}}\right)^{1 / 4}\left(\frac{M_{\mathrm{bh}}}{M_{*}}\right)^{-1 / 6}$.
This scenario implicates two important conclusions for the light curve. First, if the energy distribution is uniform the mass return rate is determined by the relation between energy and period of the orbit $\mathrm{d} M / \mathrm{d} P \propto \mathrm{d} E / \mathrm{d} P \propto P^{-5 / 3}$. Assuming the time scale of the transformation of the orbital energy into radiation is short, i.e. the luminosity of the flare follows the accretion rate and the latter is given by the mass distribution of the return times, the flux density in the flare should be

$F \propto \Delta t^{-5 / 3}$

where $\Delta t=t-t_{\mathrm{d}}$ is the time since the first passage of the pericentre. Numerical simulations have shown that this 'standard' $\Delta t^{-5 / 3}$ light curve is a good approximation, at least for later stages (Evans \& Kochanek 1989). Close to the peak luminosity the light curve can be substantially shallower (Lodato et al. 2008).

As another consequence, the time $\Delta t_{0}=t_{0}-t_{\mathrm{d}}$ the mosttightly bound material needs to fall back to $R_{\mathrm{p}}$ is directly related to the mass of the black hole

$\frac{\Delta t_{0}}{10^{-4} \mathrm{yr}} \sim \beta^{-3}\left(\frac{M_{\mathrm{bh}} R_{*}^{3}}{M_{*}^{2} k^{3}}\right)^{1 / 2}, \beta \equiv \frac{R_{\mathrm{p}}}{R_{\mathrm{t}}}$

with $M_{\mathrm{bh}}, M_{*}, R_{*}$ in solar units; $k$ depends on the spin-up state of the star with $k \sim 3$ for the likely case that the star is spun up to near break-up spin and $k \sim 1$ if spin-up is negligible.

In what follows, we shall check whether the total energy in the outburst of the Sharov 21 light curve, the decline of the outburst, and the time scales are consistent with the TDE scenario. The energy release in the flare is related to the mass of the star,

$E_{\text {flare }}=\eta f_{\text {acc }} M_{*} c^{2}$,

where $\eta$ is the efficiency of converting mass to radiated energy and $f_{\text {acc }}$ is the fraction of mass of the star accreted to the black hole. For simplicity we assume $\eta f_{\text {acc }}=0.1$, which might be quite high (see Li et al. 2002) but is not implausible, namely for $f_{\text {acc }} \sim 0.5$ following Rees (1988) and $\eta \sim 0.1 \ldots 0.4$ depending on the spin of the black hole. $E_{\text {flare }}$ is obtained by integrating the bolometric luminosity over the flare where $L_{\text {bol }}(t)$ is computed from the monochromatic flux density, $F_{v \text {,flare }}(t)=F_{v, \mathrm{obs}}(t)-\bar{F}_{v, \mathrm{gs}}$, with $F_{v, \text { obs }}(t)$ and $\bar{F}_{v, \mathrm{gs}}$ in the restframe. A black body spectrum is assumed with $T_{\text {eff }}$ as a free parameter. The lowest possible stellar mass is $M_{*} \sim 6 M_{\odot}\left(E_{\text {flare }} \sim 2 \times 10^{54} \mathrm{erg}\right)$ corresponding to $T_{\text {eff }} \sim 2 \times 10^{4} \mathrm{~K}$ where the black body spectrum peaks in the B band, i.e., at $\lambda=4400 \AA /(1+z)$ restframe. For such a spectrum a colour index $B-R \sim 0.5 \mathrm{mag}$ is expected after foreground reddening, which is in line with $B-R$ observed for Sharov 21 in the flare (Fig. 3).

With $T_{\text {eff }}, L_{\mathrm{bol}}$, and $M_{*}$ fixed, the stellar radius is constrained by Eq. (3) for a given black hole mass $M_{\mathrm{bh}}$. In Fig. 10 (top), the resulting $\left(M_{*}, R_{*}\right)$ combinations are compared with the stellar models from Salasnich et al. (2000). For $M_{\mathrm{bh}} \sim(1 \ldots 10) \times$ $10^{8} M_{\odot}$ we find $M_{*} \sim(10 \pm 3) M_{\odot}$ and $R_{*} \sim(200 \pm 100) R_{\odot}$ which excludes main-sequence stars but not giants. For such giants, the tidal radius is clearly out of the gravitation radius, $R_{\mathrm{S}}$, of the black hole (Fig. 10, middle), whereas main-sequence stars of this mass range are again excluded as $R_{\mathrm{t}}<R_{\mathrm{S}}$.

In order to check whether the decline from the maximum follows the 'standard' $\Delta t^{-5 / 3}$-law we perform linear regressions of $\log F_{v \text {,flare }}$ as a function of $\log \Delta t$ (Fig. 11). Whereas the onset of the flare at $t_{0}$ is defined by the strong increase in the light curve at JD 2448897 , the beginning of the tidal disruption, $t_{\mathrm{d}}$, has to be considered as a free parameter. The resulting slope 

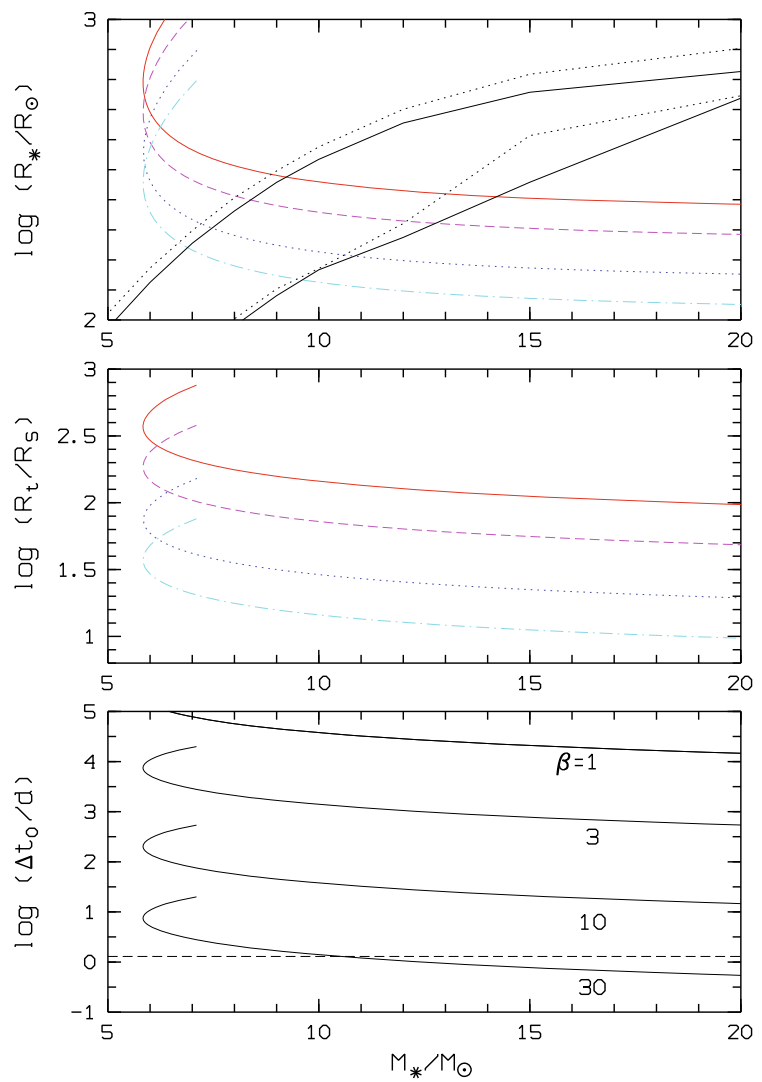

Fig. 10. Stellar radius $R_{*} / R_{\odot}$ (top), ratio of tidal radius and gravitational radius $R_{\mathrm{t}} / R_{\mathrm{S}}$ (middle), and return time $\Delta t_{0}$ in days (bottom) as a function of the mass $M_{*}$ of the tidally disrupted star for $M_{\mathrm{bh}}=1,2,5,10 \times 10^{8} M_{\odot}$ (top to bottom). In the upper panel, the curves increasing from left to right are the $R_{*}-M_{*}$ relations for the stellar models from Salasnich et al. (2000) for the base (bottom) and the top (top) of the red giant branch, respectively, for solar metallicity (dotted: solar abundance ratios, solid: $\alpha$ enhanced abundances). The dashed horizontal line in the bottom panel marks $\Delta t_{0}$ from the observed light curve (Fig. 11).

$\gamma=\mathrm{d} \log F_{v \text {,flare }} / \mathrm{d} \log \Delta t$ depends on $\Delta t_{0}$ with $\gamma \sim-1.53-0.1 \Delta t_{0}$ (days). The observed light curve is reasonably fitted by a power law with $\gamma=-5 / 3$ for $\Delta t_{0, \mathrm{obs}} \sim 1.3$ days and is shallower at the beginning of the burst, as predicted by Lodato et al. (2008).

The bottom panel of Fig. 10 shows the fallback time $\Delta t_{0}$ as a function of $M_{*}$ for different values of the penetration factor $\beta$. Note that $\Delta t_{0}$ from Eq. (5) is independent of $M_{\mathrm{bh}}$ because $R_{*}^{3} \propto M_{\mathrm{bh}}^{-1}$ for given $\left(T_{\mathrm{eff}}, L, M_{*}\right)$ (Eq. (3)). The short observed return time requires $\beta \sim 30$ for $M_{*}=10 M_{\odot}$. With $R_{\mathrm{p}} / R_{\mathrm{S}}=(4.8,2.4,1.0,0.5)$ for $M_{\mathrm{bh}}=(1,2,5,10) 10^{8} M_{\odot}$ and $M_{*} \sim 8 \ldots 12 M_{\odot}$, the fundamental condition $R_{\mathrm{p}}>R_{\mathrm{S}}$ is matched for $M_{\mathrm{bh}}<510^{8} M_{\odot}$. Guillochon et al. (2009) present highly resolved three-dimensional simulations of a tidally disrupted $1 M_{\odot}$ solar-type star approaching a $10^{6} M_{\odot}$ black hole with $\beta=7$, i.e. $R_{\mathrm{p}} / R_{\mathrm{S}} \sim 3$. Their results deviate from the case $\beta=1$ used in previous simulations. In particular, the simulated light curve shows a phase of smooth increase before the abrupt rise to the maximum, which is qualitatively in agreement with the behaviour of Sharov 21 (Fig. 2).

The TDE scenario for the flare of Sharov 21 is supported by (1) total energy in the outburst, (2) the shape of the light curve, and (3) the time scale of the event. However, it must be noticed that the standard TDE theory refers to inactive black holes. The presence of a massive, self-gravitating accretion disk is expected to have a significant effect on the dynamics of the
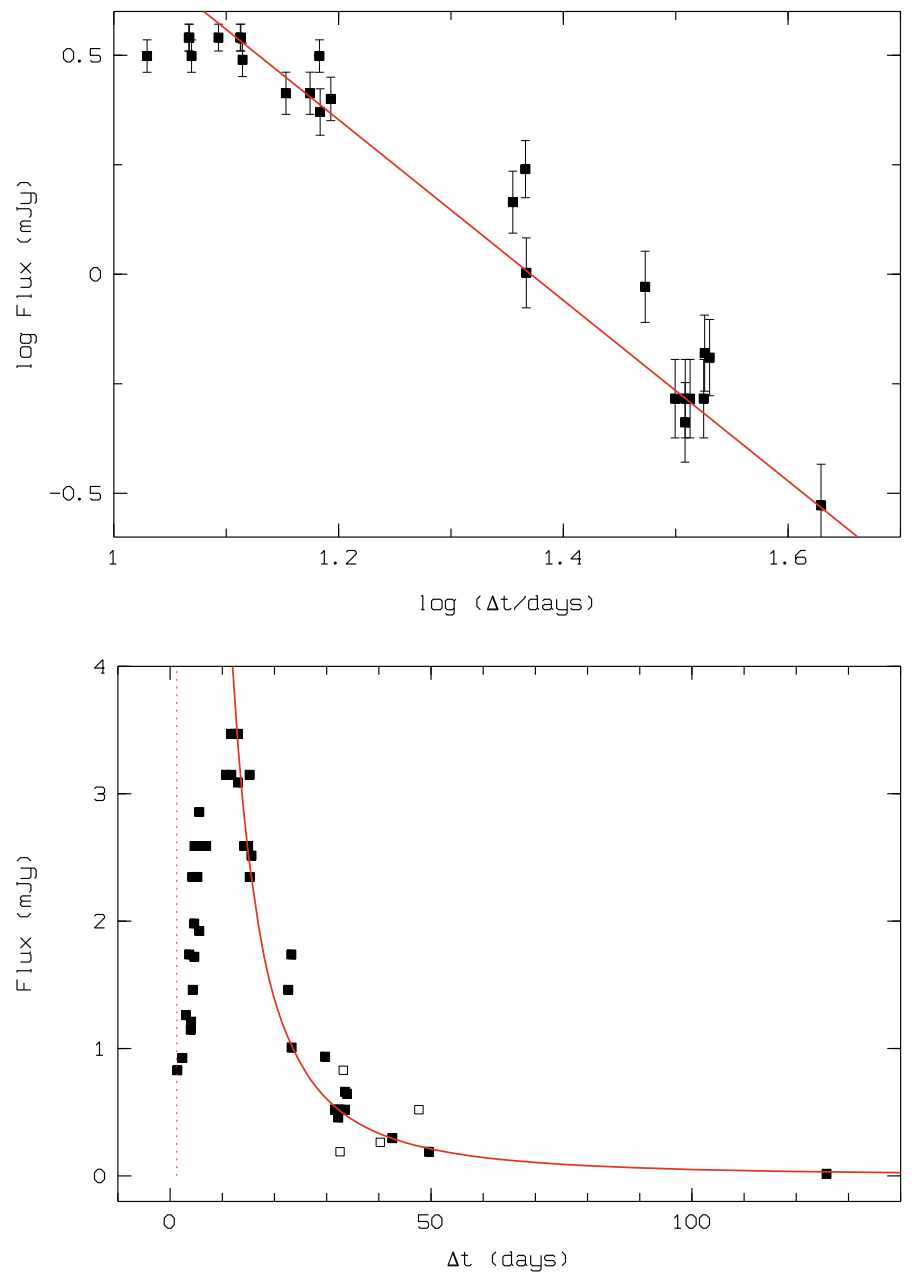

Fig. 11. Top: linear regression of the decline from the maximum in the double-logarithmic presentation of the light curve in the quasar restframe for the $\Delta t_{0}$ yielding a slope of $\gamma=-5 / 3$. Bottom: fit of the light curve in linear presentation by the result from the regression in the top panel (open symbols: uncertain data; no error bars for lucidity). The vertical dotted line marks the onset of the flare at $t_{0}$.

stellar tidal debris. Moreover, the description of the stellar orbit for an encounter with the pericentre at only a few $R_{\mathrm{S}}$ requires a fully general-relativistic treatment which is, however, beyond the scope of this paper.

One may argue that the probability for the capture of a $\sim 10 M_{\odot}$ giant star by a supermassive black hole is very low. Though this is certainly true, we emphasize that Sharov 21-like quasar flares are obviously extremely rare. Moreover it is worth mentioning (1) the high star formation rates and (2) the high fraction of massive stars in quasar host galaxies. It has been suggested for a long time that a luminous AGN phase is accompanied with or follows after an intense starburst, especially when both kinds of activity are induced by a galaxy merger (e.g., Sanders et al. 1988; Granato et al. 2004; Springel et al. 2005). Lutz et al. (2008) derived strong evidence of the presence of intense star formation (up to $\sim 3000 M_{\odot} \mathrm{yr}^{-1}$ ) from Spitzer PAH detections in 12 type 1 quasar host galaxies at $z \sim 2$. Also from Spitzer IRS observations, Shi et al. (2009) derived star formation rates on the level of luminous infrared galaxies $\left(10 \ldots 100 M_{\odot} \mathrm{yr}^{-1}\right)$ for a complete sample of 57 type-1 quasar hosts at $z \sim 1$. This high star formation activity appears to be concentrated in the circumnuclear region. In nearby Seyfert galaxies nuclear starbursts have been observed within $<100 \mathrm{pc}$ 
from the AGN (e.g., Davies et al. 2007; Watabe et al. 2008). It is likely that more luminous AGNs are accompanied by more luminous starbursts (Kawakatu \& Wada 2008). The 'radius of influence' of the black hole is $R_{\mathrm{i}}=G M_{\mathrm{bh}} / \sigma_{*}^{2}$, where $\sigma_{*}$ is the velocity dispersion of the surrounding stellar population. The $M_{\mathrm{bh}}-\sigma_{*}$ relation from Merritt \& Ferrarese (2001) yields $R_{\text {in }} \sim 12\left(M_{\mathrm{bh}} / M_{\odot}\right)^{0.58} \sim 30$ pc for $M_{\mathrm{bh}} \sim 5 \times 10^{8} M_{\odot}$. The typical distance a $10 M_{\odot}$ giant can travel with a velocity $v=\sigma_{*}$ during its lifetime is $\sim 1 \mathrm{kpc}$. (Note that the $M_{\mathrm{bh}}-\sigma_{*}$ relationship is not a strong function of redshift up to $z \sim 3$; Shields et al. 2003.)

Moreover, the central region of a galaxy provides a peculiar environment for star formation as is indicated by the high number of supergiants in our Galactic centre (e.g., Krabbe et al. 1991; Najarro et al. 1994; Martins et al. 2007; Mauerhahn et al. 2007) with nearly a hundred massive stars within the central parsec. Strong tidal forces, mass segregation, and other peculiar conditions may result in a relatively high Jeans mass for collapsing cloud cores (Morris \& Serabyn 1996) and in a top-heavy initial mass function (Figer et al. 2002; Paumard et al. 2006; Bartko et al. 2009).

Gopal-Krishna et al. (2008) argue that the gas from TDEs may be a major factor for the abortion of radio jets in quasars. If the radio loudness is related to $M_{\mathrm{bh}}$, as seems to be indicated by observations, the radio-loudness dichotomy can be explained by the existence of a critical black hole mass for the tidal disruption of solar type stars. As noted by these authors, the tidal disruption of giant stars is then required to explain the association of a few radio-quiet quasars with black holes of high masses.

\subsection{Other interpretations}

In the pioneering work of Cannon et al. (1968, 1971), the class of quasars with variability amplitudes of the order of one magnitude and with often rapid fluctuations were called optically violently variables (OVVs). OVV AGNs are all radio-loud and their strong variability is believed to be due to relativistic beaming (Gaskell \& Klimek 2003, and references therein). According to the current zoology of AGN types, OVVs constitute a subtype of blazars, i.e. of jet-dominated active galaxies viewed close $\left(\sim 15^{\circ}\right)$ to the axis of a relativistic jet (Urry \& Padovani 1995). Viewing the jet almost directly head-on results in a great magnification of variations in the flux from the jet. Blazars vary dramatically on a wide range of time scales from hours to years. In this context, the colour dependence of the variability found for the two strongest variable OVV quasars from the SDSS S82 (Sect. 4.1) can be easily understood assuming that the spectral energy distribution is dominated by the synchrotron peak in the radio and infrared. This is the case for low-peaked BL Lac objects and is typical also for flat-spectrum radio quasars: When the synchrotron emission from the jet makes a significant contribution which increases with wavelength, the optical spectrum becomes redder when the jet is brighter and the variability is stronger at longer wavelengths. Here we argue that Sharov 21 is not an OVV quasar in the "classical" sense: (1) The strong flux variation is limited to one single event, there is no evidence of strong variability on a wide range of time scales. (2) The spectral behaviour of the variability is typical of radio-quiet quasars (Sect. 4.1). (3) Sharov 21 is not radio-loud.

Variability of non-blazar AGNs has been attributed to various processes like oscillations in the accretion flow (Igumenshchev \& Abramovicz 1999) or in the jet (Hughes et al. 1998). Both models produce quasi-periodic features in the light curve where the maxima have approximately the same widths as the intervals in between, which is contrary to what we see in Sharov 21. Avalanche flows in the accretion disk provide another explanation (Tekeuchi et al. 1995). The profile of a single flare in simulated light curves (Kawaguchi et al. 1998) is characterized by a gradual increase in brightness followed by a sudden decline. It is not clear whether the rapid increase to the maximum can be explained by such a model.

An alternative explanation of AGN variability is based on the superposition of uncorrelated events such as supernova explosions (Terlevich 1992), star collisions (Courvoisier et al. 1996; Torricelli-Ciamponi et al. 2000), or colliding clumps in a clumpy accretion flow (Courvoisier \& Türler 2005). First, we compare the energy in the flare of Sharov 21 with the radiated energy of supernovae. SN 2006gy has been considered the most luminous supernova with a total radiated energy $E_{\mathrm{SN}, \mathrm{rad}} \sim 10^{51} \mathrm{erg}$ (Smith et al. 2007; Ofek et al. 2007). For the flare of Sharov 21 we estimate $E_{\text {flare }} \sim 10^{54 \ldots 55} \mathrm{erg}$, dependent on the spectral energy distribution, hence $E_{\text {flare }} \sim 10^{3 \ldots .4} E_{\mathrm{SN} \text {,rad,max }}$. It was the aim of the collision scenario to explain both the ultraviolet emission and its variability. As a modified version, such events could be rare and contribute flares to a continuum source the emission and variability of which are dominated by other processes. In fact, the star collision scenario is distantly related to the TDE model. The maximum amplitudes of the flares in the light curves presented by Torricelli-Ciamponi et al. are much too low compared to Sharov 21. However it remains to be tested if the model parameters can be adjusted to fit our light curve.

In another class of models strong flares are explained by processes in binary black holes. Lehto \& Valtonen (1996) suggest a model where they associate the quasi-periodic outbursts of the OVV quasar OJ 287 to the times when the secondary crosses the accretion disk of the primary black hole. When this happens the secondary pierces a channel through the disk, the gas is heated in the channel, flows out, and radiates strongly. The model was demonstrated to be successful for OJ 287 (Valtonen et al. 2008). Other models attribute radiative outbursts to the tidal perturbation of the accretion flow due to the secondary (Sillanpää et al. 1988), or the sweeping of a processing relativistic beam across the line of sight, in analogy to SS 433 (Katz 1997; Liu \& Chen 2007). OJ 287 is the best-studied quasar with a quasi-periodicity in the light curve. Its outbursts come in $12 \mathrm{yr}$ intervals corresponding to $9 \mathrm{yr}$ in the restframe. Our light curve covers, in the restframe, only $\sim 10 \mathrm{yr}$ before the outburst and $\sim 5 \mathrm{yr}$ after. If orbital time scales longer than about one decade are not atypical of supermassive black hole binaries, it cannot be excluded that the flare of Sharov 21 is related to such a process. It would be important to find more historical observations in plate archives which yield an extension of the light curve further back. This however is much more difficult than for OJ 287 which is $\sim 5$ mag brighter than Sharov 21.

Finally, we briefly mention the possibility that the quasar was by chance superposed by a nova in M31. This hypothesis was tested already by Sharov et al. (1998). From the measurement of the position before, during, and after the flare they find that the derived coordinates are in excellent agreement with each other and conclude that the flaring object is "coincident with the star that was visible at its place in 1982-1997".

\section{Summary and conclusions}

Sharov 21, originally discovered by Nedialkov et al. (1996), was previously classified as a remarkable nova in M31 (Sharov et al. 1998). Here we have shown that it is a typical type 1 quasar at $z=2.109$ seen through the disk of M31 and showing an 
exceptionally strong UV flare. We created a significantly improved long-term light curve based on archival data, data from the literature, and targetted new observations. Compared to the original data given by Sharov et al., the new light curve has a $\sim 20 \mathrm{yr}$ longer baseline and a better sampling. Altogether, more than $10^{3}$ single exposures from 15 wide field telescopes are included resulting in detections at 221 epochs from 1948 to 2009, with a relatively good time coverage after 1961 . The data material is completed by a large number of observations without detection of the quasar but with useful upper brightness limits for the time interval from 1900 to 2009 . Based on this data, we subdivide the light curve in two phases: a rather quiet ground state with $\bar{B} \sim 20.5$ for at least $98 \%$ of the time and a strong outburst in 1992 with an increase of the UV flux by a factor 20 .The variability in the ground state does not significantly differ from that of other radio-quiet quasars of comparable redshift and luminosity. A black hole mass of $5 \times 10^{8} M_{\odot}$ is estimated from the C IV line, corresponding to an Eddington ratio of $\sim 0.6$ for the ground state. By the comparison with $\sim 8000$ quasars in the stripe 82 of the SDSS on a 7 yr-baseline (Bramich et al. 2008) and more than 300 VPMS quasars (Meusinger et al. 2002, 2003) with light curves having a time-baseline comparable to Sharov 21 we have demonstrated that the strong UV flare of Sharov 21 is very unusual for radio-quiet high-redshift quasars. We conclude that such a rare feature is the result of a rare event. As such we suggest two scenarios: (1) gravitational microlensing due to a star in M31 and (2) a tidal disruption event (TDE) of a star close to the supermassive black hole of the quasar.

In the TDE scenario, the total energy in the outburst can be explained by the disruption of a $\sim 10 M_{\odot}$ giant star if we "optimistically" assume that half of the disrupted star is accreted to the black hole and $\sim 20 \%$ of the accreted energy can be radiated away. The flare profile shows a sudden increase to the maximum followed by a decline which is reasonably fitted by the $\Delta t^{-5 / 3}$ power law predicted by the standard TDE model. The short time span between the beginning of the tidal disruption and the beginning of the flare, $\Delta t_{0} \sim 1.3$ days, requires an ultra-close encounter with $\beta \sim 30$ corresponding to a stellar orbit with the pericentre at only a few $R_{\mathrm{S}}$. The present study has not taken general-relativistic effects into account. Moreover, it is unclear how the TDE scenario is affected by the presence of a massive accretion disk. Microlensing by a star in M 31 is a plausible alternative explanation. Though the detection of a microlensed quasar behind M 31 over half a century is not unlikely, high-amplification events corresponding to the flare of Sharov 21 are very rare. We apply the point source-point lens approximation to model the light curve. Assuming a transverse velocity of $300 \mathrm{~km} \mathrm{~s}^{-1}$, an acceptable fit is achieved for a low-mass binary with $0.3 M_{\odot}$ and $0.1 M_{\odot}$. The observed light curve of Sharov 21 is roughly fitted by either of the two scenarios, but more detailed modelling is necessary to decide if the flare can be reproduced accurately. Finally, we cannot exclude that the flare is part of a quasi-periodic activity similar to OJ 287 (Valtonen et al. 2008) on an intrinsic time scale of $\gtrsim 10 \mathrm{yr}$. The remarkable quasar Sharov 21 obviously merits further efforts, both for the completion of the light curve and for its modelling.

Finally, we notice that there is an interesting application of Sharov 21-like flares. Projects like PTF, Pan-STARRS, and LSST will probably discover several such events. Due to their enormous luminosity and long time scale in the observer frame, such flares can provide background light sources for intervening matter and create thus interesting opportunities for highresolution spectroscopy of matter at large distances. This argument has been presented by Quimby et al. (2009) in the context of the brightest supernovae from the PTF but holds even more for quasar flares comparable to that one of Sharov 21.

Acknowledgements. The anonymous referee is greatly acknowledged for his constructive criticism which helped to improve the paper. We thank S. Komossa for helpful comments on an earlier draft version of this paper and A. Kann, S. Klose, M. Röder, S. Schulze, and B. Stecklum for contributing observations with the Tautenburg Schmidt CCD camera. We are grateful to M. Demleitner, Astronomisches Rechen-Institut, Heidelberg, for installing and managing the HDAP data base as part of GAVO, to G. Langer and L. Siegwald, LSW HD, for scanning the Heidelberg and Calar Alto plates, and to O. Stahl, LSW $\mathrm{HD}$, for manifold support, particularly with data handling. The HDAP project is financed by the Klaus Tschira Foundation, Heidelberg under contract no. 00.071.2005. The Tautenburg plate scanner was financially supported by the Deutsche Forschungsgesellschaft under grants Me1350/3 and Me1350/8. M.H. acknowledges support from the BMWI/DLR, FKZ 50 OR 0405. This research draws upon data provided by Philip Massey as distributed by the NOAO Science Archive. NOAO is operated by the Association of Universities for Research in Astronomy (AURA), Inc. under a cooperative agreement with the National Science Foundation. We make also use of data obtained from the Isaac Newton Group Archive which is maintained as part of the CASU Astronomical Data Centre at the Institute of Astronomy, Cambridge, and used observations taken with the Canada-France-Hawaii Telescope (CFHT) which is operated by the National Research Council of Canada, the Institut National des Sciences de l'Univers of the Centre National de la Recherche Scientifique of France, and the University of Hawaii. Some of the data presented in this paper were obtained from the Multimission Archive at the Space Telescope Science Institute (MAST). STScI is operated by the Association of Universities for Research in Astronomy, Inc., under NASA contract NAS5-26555. Support for MAST for non-HST data is provided by the NASA Office of Space Science via grant NAG57584 and by other grants and contracts.

\section{References}

Abramovicz, M. A., Szuszkiewicz, E., \& Wallinder, F. 1989, in Theory of Accretion Discs, ed. F. Meyer, W. J. Duschl, J. Frank \& E. Meyer-Hofmeister (London: Kluwer Academic Publications), 141

Alksnis, A., Balklavs, A., Eglitis, I., \& Paupers, O. 1998, Baltic Astron., 7, 653 Ayal, S., Livio, M., \& Piran, T. 2000, ApJ, 545, 772

Barbieri, C., Omizzolo, A., \& Rampazzi, F. 2003, Mem. S.A.It., 74, 430

Barmby, P., Huchra, J., Brodie, J., et al. 2000, AJ, 119, 727

Bartko, H., Martins, F., Trippe, S., et al. 2010, ApJ, 708, 834

Bauer, A., Baltay, C., Coppi, P., et al. 2009, ApJ, 705, 46

Bertin, E., \& Arnouts, S. 1996, A\&AS 117, 393

Bogdanović, T., Eracleous, M., Mahadevan, S., et al. 2004, ApJ, 610, 707

Bramich, D. M., Vidrih, S., Wyrzykowski, L., et al. 2008, MNRAS, 386, 887

Braun, R. 1990, ApJS, 72, 761

Brunzendorf, J., \& Meusinger, H. 1999, A\&AS, 139, 141

Cannon, R. D., Penston, M. V., \& Penston, M. J. 1968, Nature, 217, 340

Cannon, R. D., Penston, M. V., \& Brett, R. A. 1971, MNRAS, 152, 79

Cappelluti, N., Ajello, M., Rebusco, P., et al. 2009, A\&A, 495, L9

Chae, K. H., Turnshek, D. A., Schulte-Ladbeck, R. E., et al. 2001, ApJ, 561, 653

Chang, K., \& Refsdal, S. 1979, Nature, 282, 261

Chartas, G., Eracleous, M., Algol, E., et al. 2004, ApJ, 606, 78

Chen, X., Liu, F. K., \& Magorrian, J. 2008, ApJ, 676, 54

Cid Fernandes, R., Terlevich, R., \& Aretxaga, I. 1997, MNRAS, 289, 318

Corbett, E. A., Croom, S. M., Boyle, B. J., et al. 2003, MNRAS, 343, 705

Courvoisier, T. J.-L., \& Türler, M. 2005, A\&A, 444, 417

Courvoisier, T. J.-L., Paltani, S., \& Walter, R. 1996, 308, A\&A 308, L17

Cristiani, S., Trentini, S., La Franca, F., \& Andreani, P. 1997, A\&A, 321, 123

Cutri, R. M., Wiśniewski, W. Z., Rieke, G. H., \& Lebofsky, M. J. 1985, ApJ, 296, 423

Davies, R. I., Mueller Sánchez, F., Genzel, R., et al. 2007, ApJ, 671, 1388

de Vaucouleurs, G., de Vaucouleurs, A., Corwin, H. G., et al. 1991, Third

Reference Catalogue of Bright Galaxies (New York: Springer)

Di Clemente, A., Giallongo, E., Natali, G., et al., 1996, ApJ, 463, 466

Dong, X., Wang, J., Wang, T., et al. 2009, [arXiv:0903.5020], unpublished

Donley, J. L., Brandt, W. N., Eracleous, M. J., \& Boller, Th. 2002, AJ, 124, 1398

Edelson, R. A., Krolik, J. H., \& Pike, G. F. 1990, ApJ, 359, 86

Eigenbrod, A., Courbin, F., Dye, S., et al. 2006, A\&A, 451, 747

Elvis, M., Wilkes, B., McDowell, J., et al. 1994, ApJS, 95, 1

Esquej, R. D., Saxton, R. D., Freyberg, M. J., et al. 2007, A\&A, 462, L49

Esquej, P., Saxton, R. D., Komossa, S., et al. 2008, A\&A, 489, 543

Evans, C. R., \& Kochanek, C. S. 1989, ApJ, 346, L13

Fan, Z., Ma, J., de Grijs, R., \& Zhou, X. 2008, MNRAS, 385, 1973

Ferguson, A. M. N., Irwin, M. J., Ibata, R. A., et al. 2002, AJ, 124, 1452

Figer, D. F., Najarro, F., Gilmore, D., et al. 2002, ApJ, 581, 202 
Froebrich, D., \& Meusinger, H. 2000, A\&AS, 145, 229

Gaskell, C. M., \& Klimek, E. S. 2003, Astron. Astrophys. Trans., 22, 661 Gavignaud, I., Wisotzki, L., Bongiorno, A., et al. 2008, A\&A, 492, 637

Geehan, J. J., Fardal, M. A., Babul, A., \& Guhathakurta, P. 2006, MNRAS, 366, 996

Gelfand, J. D., Lazio, T. J. W., \& Gaensler, B. M. 2004, ApJS, 155, 89

Gelfand, J. D., Lazio, T. J. W., \& Gaensler, B. M. 2005, ApJS, 159, 242

Gezari, S., Martin, D. C., Milliard, B., et al. 2006, ApJ, 653, L25

Gezari, S., Basa, S., Martin, D. C., et al. 2008, ApJ, 676, 944

Gopal-Krishna, Mangalam A., \& Wiita, P. J. 2008, ApJ, 680, L13

Guillochon, J., Ramirez-Ruiz, E., Rosswog, S., \& Kasen, D. 2009, ApJ, 705, 844

Granato, G. L., de Zotti, G., Silva, L., et al. 2004, ApJ, 600, 580

Hatano, K., Branch, D., Fisher, A., \& Starrfield, S. 1997, ApJ, 487, L45

Hawkins, M. R. S. 1993, Nature, 366, 242

Hawkins, M. R. S., \& Véron, P. 1993, MNRAS, 260, 202

Henze, M., Meusinger, H., \& Pietsch, W. 2008, A\&A, 477, 67

Hills, J. G. 1975, Nature, 254, 27

Honma, F., Matsumoto, R., \& Kato, S. 1991, PASJ, 43, 147

Hubble, E., 1929, ApJ, 69, 103

Hughes, P. A., Aller, H. D., \& Aller, M. F. 1998, ApJ, 503, 662

Igumenshchev, I. V., \& Abramowicz, M. A. 1999, MNRAS, 303, 309

Irwin, M. J., Webster, R. L., Hewett, P. C., et al. 1989, AJ, 98, 1989

Irwin, M. J., Ferguson, A. M. N., Ibata, R. A., et al. 2005, ApJ, 628, L105

Ivanov, P. B., \& Novikov, I. D. 2001, ApJ, 549, 467

Jackson, N., Battye, R. A., Browne, I. W. A., et al. 2007, MNRAS, 376,371

Katz, J. I. 1997, ApJ, 478, 527

Kawaguchi, T., Mineshige, S., Umemura, M., \& Turner, E. L. 1998, ApJ, 504, 671

Kawakatu, N., \& Wada, K. 2008, ApJ, 681, 73

Kollmeier, J. A., Onken, C. A., Kochanek, C. S., et al. 2006, ApJ, 648, 128

Komossa, S. 2002, in Lighthouses of the Universe, ed. M. Gilfanov, R. A. Sunyaev, \& E. Churazov (Berlin: Springer), 436

Komossa, S., \& Bade, N. 1999, A\&A, 343, 775

Komossa, S., \& Merritt, D. 2008, ApJ, 683, L21

Komossa, S., Halpern, J., Schartel, N., et al. 2004, ApJ, 603, L17

Komossa, S., Zhou, H., Rau, A., et al. 2009, ApJ, 701, 105

Koopmans, L. V. E., de Bruyn, A. G., Xauthopoulos, E., \& Fassnacht, C. D. 2000, A\&A, 356, 391

Krabbe, A., Genzel, R., Drapatz, S., \& Rotaciuc, V. 1991, ApJ, 382, L19

Kron, R. G., \& Chiu L.-T. G. 1981, PASP, 93, 397

Lacy, J. H., Townes, C. H., \& Hollenbach, D. J. 1982, ApJ, 262, 120

Lewis, G. F., \& Irwin, M. J. 1996, MNRAS, 283, 225

Li, L.-X., Narayan, R., \& Menou, K. 2002, ApJ, 576, 753

Litskii, V. V., \& Ozernoi, L. M. 1979, AZh Pis'ma, 5, 28

Liu, F. K., \& Chen, X. 2007, ApJ, 671, 1272

Lehto, H. J., \& Valtonen, M. J. 1996, ApJ, 460, 207

Lodato, G., King, A. R., \& Pringle, J. E. 2009, MNRAS, 392, 332

Loeb, A., \& Narayan, R. 2008, MNRAS, 386, 2221

Lutz, D., Sturm, E., Tacconi, L. J., et al. 2008, ApJ, 684, 853

Magorrian, J., \& Tremaine, S. 1999, MNRAS, 309, 447

Majewski, S. R., Munn, J. A., Kron, R. G., et al., 1991, in The space distribution of quasars, ed. D. Crampton (San Francisco), ASP Conf. Ser., 21, 66

Mao, S. 2008, in The 12th International Conference and ANGLES Microlensing Workshop, ed. E. Kerins, S. Mao, N. Rattenbury, \& L. Wyrzykowski, published online at SISSA Proceedings of Science, 2

Margon, B., \& Deutsch, E. W. 1997, PASP, 109, 673

Massey, P., Olson, K. A. G., Hodge, P. W., et al. 2006, AJ, 131, 2478

Martins, F. Genzel, R., Hillier, D. J., et al. 2007, A\&A, 468, 233

Mauerhahn, J. C., Muno, M. P., \& Morris, M. 2007, ApJ, 662, 574

Menou, K., \& Quataert, E. 2001, ApJ, 562, L137

Merritt, D., \& Ferrarese, L. 2001, ApJ, 547, 140

Meusinger, H., Scholz, R.-D., Irwin, M., \& Lehmann, H. 2002, A\&A, 392, 851

Meusinger, H., Brunzendorf, J., \& Laget, M. 2003, Astron. Nachr., 324, 474

Miloslavljević, M., Merritt, D., \& Ho, L. C. 2006, ApJ, 652, 120

Monet, D. G., Levine, S. E., Canzian, B., et al. 2003, AJ, 125, 984

Morris, M., \& Serabyn, E. 1996, ARA\&A, 34, 645

Najarro, F., Hillier, D. J., Kudritzki, R. P., et al. 1994, A\&A, 285, 573

Nedialkov, P. L., Kurtev, R. G., Tikhonov, N. A., \& Ivanov, G. R. 1996, Inf. Bull. Var. Stars, 4411

Ofek, E. O., Cameron, P. B., Kasliwal, M. M., et al. 2007, ApJ, 659, L13

Onken, C. A., \& Kollmeier, J. A. 2008, 689, ApJ, L13

Östman, L., Goobar, A., \& Mörtsell, E. 2006, A\&A, 450,971

Paczyński, B., 1986, ApJ, 301, 503

Paltani, S., \& Courvoisier, T. J.-L. 1994, A\&A, 291, 74

Paraficz, D., Hjorth, J., Burud, I., et al. 2006, A\&A, 455, L1
Paumard, T., Genzel, R., Martins, F., et al. 2006, ApJ, 643, 1011 Pelt, J., Schild, R., Refsdal, S., \& Stabell, R. 1998, A\&A, 336, 829 Peterson, B. M., Ferrarese, L., Gilbert, K. M., et al. 2004, ApJ, 613, 682 Phinney, E. S. 1989, in The Center of the Galaxy, ed. M. Morris (Dordrecht: Kluwer), IAU Symp., 136, 543

Pietsch, W., Fliri, J., Freyberg, M. J., et al. 2005a, A\&A, 442, 879

Pietsch, W., Freyberg, M. J., \& Haberl, F. 2005b, A\&A, 434, 483

Predehl, P., \& Schmitt, J. H. M. M. 1995, A\&A, 293, 889

Quimby, R. M., Kulkarni, S. R., Kasliwal, M. M., et al. 2010, [arXiv: 0910.0059], Nature, submitted

Racine, R. 1991, AJ, 101, 865

Rees, M. J. 1988, Nature, 333, 523

Rees, M. J. 1990, Science, 247, 817

Rengstorf, A. W., Mufson, S. L., Abad, C., et al. 2004, ApJ, 606, 741

Renzini, A., Greggio, L., di Serego-Alighieri, S., et al. 1995, Nature, 378, 39

Richards, G. T., Lacy, M., Storrie-Lombardi, L. J., et al. 2006, ApJS, 166, 470

Salasnich, B., Girardi, L., Weiss, A., \& Chiosi, C. 2000, A\&A, 361, 1023

Sandage, A. 1961, The Hubble Atlas of Galaxies (Washington D.C.: Carnegie Inst. of Washington)

Sanders, D. B., Soifer, B. T., Elias, J. H., et al. 1988, ApJ, 325, 74

Savage, B. D., \& Mathis, J. S. 1979, ARA\&A, 17, 73

Schlegel, D. J., Finkbeiner, D. P., \& Davis, M. 1998, ApJ, 500, 525

Schneider, D. P., Hall, P. B., Richards, G. T., et al. 2007, AJ, 134, 102

Schneider, P. 1993, A\&A, 279, 1

Sesar, B., Ivezić, Ž., Lupton, R. H., et al. 2007, AJ, 134, 2236

Sharov, A. S., Alksnis, A., Nedialkov, P. L., et al. 1998, Astron. Lett., 24, 445

Shen, Y., Greene, J. E., Strauss, M. A., et al. 2008, ApJ, 680, 169

Shi, Y., Rieke, G. R., Ogle, P., et al. 2010, ApJ, 703, 1107

Shields, G. A., Gebhardt, K., Salviander, S., et al. 2003, ApJ, 583, 124

Sillanpää, A., Haarala, S., Valtonen, M. J., et al. 1988, ApJ, 325, 628

Simonetti, J. H., Cordes, J. M., \& Heeschen, D. S. 1985, ApJ, 296, 46

Skrutskie, M. F., Cutri, R. M., Stiening, R., et al. 2006, AJ, 131, 1163

Sluse, D., Claeskens, J.-F., Hutsemékers, D., et al. 2007, A\&A, 468, 885

Smith, N., Li, W, Foley, R. J., et al. 2007, ApJ, 666, 1116

Sowards-Emmerd, D., Romani, R. W., Michelson, P. F., et al. 2005, ApJ, 626, 95

Springel, V., Di Matteo, T., \& Hernquist, L. 2005, MNRAS, 361, 776

Stocke, J. T., Morris, S. L., Weymann, R. J., \& Foltz, C. B. 1992, ApJ, 396, 487

Strubbe, L. E., \& Quataert, E. 2009, MNRAS, 400, 2070

Supper, R., Hasinger, G., Lewin, W. H. G., et al. 2001, A\&A, 373, 63

Syer, D., Clarke, C. J., \& Rees, M. J. 1991, MNRAS, 250, 505

Taam, R. E., \& Lin, D. N. C. 1984, ApJ, 287, 761

Takeuchi, M., Mineshige, S., \& Negoro, H. 1995, PASJ, 47, 617

Terlevich, R., Tenorio-Tagle, G., Franco, J., \& Melnick, J. 1992, MNRAS, 255, 713

Torricelli-Ciamponi, G., Foellmi, C., Courvoisier, T. J.-L., \& Paltani, S. 2000, A\&A, 358, 57

Trèvese, D., Kron, R. G., \& Bunone, A. 2001, ApJ, 551, 103

Tsvetkov, M. K., Stavrev, K. Y., Tsvetkova, K. P., et al. 1997, VizieR On-line Catalog: VI/90

Ulmer, A. 1999, ApJ, 514, 180

Ulrich, M.-H., Maraschi, L., \& Urry, C. M. 1997, ARA\&A, 35, 445

Urry, C. M., \& Padovani, P. 1995, PASP, 107, 803

Valtonen, M. J., Lehto, H. J., Nilsson, K., et al. 2008, Nature, 452, 851

Vanden Berk, D. E., Richards, G. T., Bauer, A., et al. 2001, AJ, 122, 549

Vanden Berk, D. E., Wilhite, B. C., Kron, R. G., et al. 2004, ApJ, 601, 692

Vilardell, F., Ribas, I., \& Jordi, C. 2006, A\&A, 459, 321

Véron-Cetty, M.-P. \& Véron, P. 2006, A\&A, 455, 773

Vestergaard, M., 2002, ApJ, 571, 733

Vestergaard, M., 2004, ApJ, 601, 676

Vestergaard, M., 2009, in 2007 STScI Spring Symposium Black Holes, ed.

M. Livio \& A. M. Koekemoer (Cambridge University Press), in press, [arXiv:0904.2615v1]

Vestergaard, M., \& Peterson, B. M. 2006, ApJ, 641, 689

Vietri, M., \& Ostriker, J., P. 1983, ApJ, 267, 488

Wambsganss, J., \& Paczyński, B. 1991, AJ, 102, 864

Wang, J., \& Merritt, D. 2004, ApJ, 600, 149

Warner, C., Hamann, F., \& Dietrich, M. 2003, ApJ, 596, 72

Watabe, Y., Kawakatu, N., \& Imanishi, M. 2008, ApJ, 677, 895

White, R. L., Becker, R. H., \& Gregg, M. D. 2000, ApJS, 126, 133

Wilhite, B. C., Vanden Berk, D. E., Kron, R., G., et al. 2005, ApJ, 633, 638

Wisotzki, L., Becker, T., Christensen, L., et al. 2003, A\&A, 408, 455

Wisotzki, L., Schechter, P. L., Chen, H.-W., et al. 2004, A\&A, 419, 31

Yonehara, A., Hirashita, H., \& Richter, P. 2008, A\&A, 478, 95

Zackrisson, E., Bergvall, N., Marquart, T., \& Helbig, P. 2003, A\&A, 408, 17 